\title{
Weak localization, Aharonov-Bohm oscillations and decoherence in arrays of quantum dots
}

\author{
Dmitri S. Golubev ${ }^{1}$, Andrew G. Semenov ${ }^{2}$ and Andrei D. Zaikin ${ }^{1,2}$ \\ ${ }^{1}$ Institute of Nanotechnology, Karlsruhe Institute of Technology (KIT), 76021 Karlsruhe, Germany \\ ${ }^{2}$ I.E. Tamm Department of Theoretical Physics, P.N. Lebedev Physics Institute, 119991 Moscow, Russia
}

\begin{abstract}
Combining scattering matrix theory with non-linear $\sigma$-model and Keldysh technique we develop a unified theoretical approach enabling one to non-perturbatively study the effect of electron-electron interactions on weak localization and Aharonov-Bohm oscillations in arbitrary arrays of quantum dots. Our model embraces (i) weakly disordered conductors (ii) strongly disordered conductors and (iii) metallic quantum dots. In all these cases at $T \rightarrow 0$ the electron decoherence time is found to saturate to a finite value determined by the universal formula which agrees quantitatively with numerous experimental results. Our analysis provides overwhelming evidence in favor of electron-electron interactions as a universal mechanism for zero temperature electron decoherence in disordered conductors.
\end{abstract}

\section{INTRODUCTION}

Quantum interference of electrons is a fundamentally important phenomenon which can strongly electron transport in disordered conductors $\mathrm{s}^{-3}$. . Quantum coherent effects are mostly pronounced at low temperatures in which case certain interaction mechanisms are "frozen out" and, hence, do not anymore limit the ability of electrons to interfere. However, there exists at least one mechanism, electron-electron interactions, which remains important down to lowest temperatures and may destroy quantum interference of electrons down to $T=0$. In a series of papers ${ }^{4}$ two of the present authors formulated a general theoretical formalism which allows to describe electron interference effects in the presence of disorder and electron-electron interactions at any temperature, including the limit $T \rightarrow 0$. This approach extends Chakravarty-Schmid description 22 of weak localization (WL) and generalizes Feynman-Vernon path integral influence functional technique $\mathrm{E}^{\underline{5}}$ to fermionic systems with disorder and interactions. With the aid of this technique it turned out to be possible to quantitatively explain low temperature saturation of WL correction to conductance $\delta G^{W L}(T)$ commonly observed in diffusive metallic wires 6.7 . It was demonstrated $\underline{\underline{4}}$ that this saturation effect is caused by electron-electron interactions.

It is worth pointing out that low temperature saturation of WL correction and of the electron decoherence time $\tau_{\varphi}$ (extracted from $\delta G^{W L}(T)$ or by other means) has been repeatedly observed not only in metallic wires but also in virtually any type of disordered conductors ranging from individual quantum dots ${ }^{8}$ to very strongly disordered $3 \mathrm{~d}$ structures and granular metals $\stackrel{9}{ }$. Hence, it is plausible that in all these systems we are dealing with the same fundamental effect of electron-electron interactions. In order to test this conjecture it is necessary to develop a unified theoretical description which would cover essentially all types of disordered conductors. Although the approach $\underline{4}^{4}$ is formally an exact procedure treating electron dynamics in the presence of disorder and interactions, in some cases, e.g., for quantum dots and gran- ular metals, it can be rather difficult to directly evaluate $\delta G^{W L}(T)$ within this technique.

One of the problems in those cases is that the description in terms of quasiclassical electron trajectories may become insufficient, and electron scattering on disorder should be treated on more general footing. In addition, within the approach ${ }^{4}$ disorder averaging is (can be) postponed until the last stage of the calculation which is convenient in certain physical situations. In other cases like ones studied below - it might be, in contrast, more appropriate to perform disorder averaging already in the beginning of the whole analysis. Finally, it is desirable to deal with the model which would embrace various types of conductors with well defined properties both in the long and short wavelength limits.

Below we will elaborate an alternative approach which combines the scattering matrix and Keldysh techniques with the description of electron-electron interactions in terms of quantum Hubbard-Stratonovich fields. Note that previously a similar type of approach was employed in order to describe Coulomb effects in tunnel junctions, see, e.g. ${ }^{10,11}$. Here we will describe a disordered conductor by means of an array of (metallic) quantum dots connected via junctions (scatterers) with an arbitrary distribution of transmissions of their conducting channels. This model will allow to easily crossover between the limits of granular metals and those with point-like impurities and to treat spatially restricted and spatially extended conductors within the same theoretical framework. Electron scattering on each such scatterer will be treated within the most general scattering matrix formalism ${ }^{12.13}$ adopted to include electron-electron interaction effects $14-21$. Averaging over disorder will be performed within the non-linear $\sigma$-model technique in Keldysh formulation. This method has certain advantages over the imaginary time approach since it allows to treat both equilibrium and non-equilibrium problems and also enables one to include Coulomb interaction between electrons in a straightforward manner ${ }^{22}$.

In this paper we will review and extend our analysis of weak localization effects and Aharonov-Bohm oscillations 
in systems composed of metallic quantum dots $23-27$. In Sec. 2 we will construct a theory for essentially noninteracting electrons including interaction effects only phenomenologically by introducing an effective electron dephasing time $\tau_{\varphi}$ as an independent parameter. In Sec. 3 we will develop a systematic unified analysis of the effect of electron-electron interactions on weak localization and Aharonov-Bohm oscillations in both quantum dots and extended diffusive conductors. Sec. 4 is devoted to a comparison of our results with experimental observations.

\section{WEAK LOCALIZATION IN QUANTUM DOT ARRAYS}

\section{A. The model and basic formalism}

Let us consider a 1d array of connected in series chaotic quantum dots (Fig. 11). Each quantum dot is characterized by its own mean level spacing $\delta_{n}$. Adjacent quantum dots are connected via barriers which can scatter electrons. Each such scatterer is described by a set of transmissions of its conducting channels $T_{k}^{(n)}$ (here $k$ labels the channels and $n$ labels the scatterers). Below we will ignore spin-orbit scattering and focus our attention on the case of $1 d$ arrays. If needed, generalization of our analysis to systems of higher dimensions can be employed in a straightforward manner ${ }^{23}$.

An effective action $S[\check{Q}]$ of an array depicted in Fig. 1 depends on the fluctuating $4 \times 4$ matrix fields 19,23 $\check{Q}_{n}\left(t_{1}, t_{2}\right)$ defined for each of the dots $(n=1, \ldots, N-1)$. Each of these fields is a function of two times $t_{1}$ and $t_{2}$ and obeys the normalization condition

$$
\check{Q}_{n}^{2}=1
$$

The action of an array can be represented as a sum of two terms

$$
i S[\check{Q}]=i S_{d}[\check{Q}]+i S_{t}[\check{Q}]
$$

The first term, $i S_{d}[\check{Q}]$, describes the contribution of bulk parts of the dots. This term reads

$$
i S_{d}[\check{Q}]=\sum_{n=1}^{N-1} \frac{\pi}{\delta_{n}} \operatorname{Tr}\left[\frac{\partial}{\partial t} \check{Q}_{n}-\alpha_{n} H^{2}\left(\left[\check{A}, \check{Q}_{n}\right]\right)^{2}\right]
$$

Here $H$ is an external magnetic filed, $\alpha_{n}=$ $b_{n}\left(e^{2} / \hbar^{2} c^{2}\right) v_{F} d_{n}^{2} \min \left\{l_{e}, d_{n}\right\}, b_{n}$ is a geometry dependent numerical prefactor ${ }^{13}, d_{n}$ is the size of $n$-th dot, $l_{e}$ is the elastic mean free path in the dot, and $\check{A}$ is $4 \times 4$ matrix:

$$
\check{A}=\left(\begin{array}{cccc}
1 & 0 & 0 & 0 \\
0 & -1 & 0 & 0 \\
0 & 0 & 1 & 0 \\
0 & 0 & 0 & -1
\end{array}\right)
$$

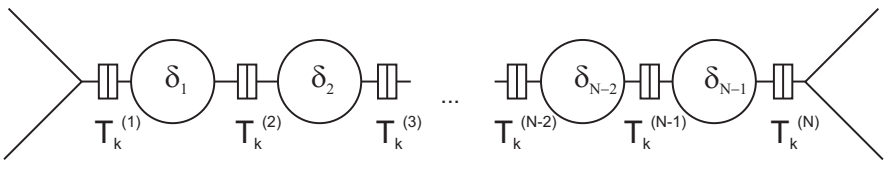

FIG. 1: 1d array of $N-1$ quantum dots coupled by $N$ barriers. Each quantum dot is characterized by mean level spacing $\delta_{n}$. Each barrier is characterized by a set of transmissions of its conducting channels $T_{k}^{(n)}$.

The second term in Eq. (2), $i S_{t}[\check{Q}]$, describes electron transfer between quantum dots. It has the form 28

$i S_{t}[\check{Q}]=\frac{1}{2} \sum_{n=1}^{N} \sum_{k} \operatorname{Tr} \ln \left[1+\frac{T_{k}^{(n)}}{4}\left(\left\{\check{Q}_{n-1}, \check{Q}_{n}\right\}-2\right)\right]$

Note that here the magnetic field $H$ is included only in the term (3) describing the quantum dots while it is ignored in the term (5). Usually this approximation remains applicable at not too low magnetic fields.

An equilibrium saddle point configuration $\check{\Lambda}\left(t_{1}-t_{2}\right)$ of the matrix field $\check{Q}\left(t_{1}, t_{2}\right)$ depends only on the time difference and has the form

$$
\check{\Lambda}(t)=\int \frac{d E}{2 \pi} e^{-i E t}\left(\begin{array}{crrc}
-1 & 0 & 0 & 0 \\
0 & 1 & 0 & 0 \\
g^{K}(E) & 0 & 1 & 0 \\
0 & -g^{K}(E) & 0 & -1
\end{array}\right),
$$

where $g^{K}(E)=2\left[1-2 f_{F}(E)\right]=2 \tanh (E / 2 T)$. This choice of the saddle point corresponds to the following structure of the $4 \times 4$ matrix Green function $\check{G}$ :

$$
\check{G}=\left(\begin{array}{cccc}
G^{A} & 0 & 0 & 0 \\
0 & \mathcal{T} G^{A *} \mathcal{T} & 0 & \\
-G^{K} & 0 & G^{R} & 0 \\
0 & \mathcal{T} G^{K *} \mathcal{T} & 0 & \mathcal{T} G^{R *} \mathcal{T}
\end{array}\right)
$$

Here we defined the time inversion operator $\mathcal{T}$ :

$$
\mathcal{T} f(t)=f\left(t_{f}-t\right),
$$

where $t_{f}$ will be specified later. Note that the function $\check{G}$ in Eq. (7), defined for a given disorder configuration, should be contrasted from the Green function

$$
\check{G}_{Q}=\left[i \frac{\partial}{\partial t}+\frac{\nabla^{2}}{2 m}+\frac{i}{2 \tau_{e}} \check{Q}^{-1}\right.
$$

defined for a given realization of the matrix field $\check{Q}$. In Eq. (9) we also introduced the electron elastic mean free time $\tau_{e}$.

\section{B. Gaussian approximation}

In order to evaluate the WL correction to conductance we will account for quadratic (Gaussian) fluctuations of 
the matrix field $\check{Q}_{n}$. This approximation is always sufficient provided the conductance of the whole sample exceeds $e^{2} / h$, in certain situations somewhat softer applicability conditions can be formulated. Expanding in powers of such fluctuations we introduce the following parameterization

$$
\begin{aligned}
& \check{Q}_{n}=e^{i \check{W}_{n}} \check{\Lambda}^{-i \check{W}_{n}} \\
& =\check{\Lambda}+i\left[\check{W}_{n}, \check{\Lambda}\right]+\check{W}_{n} \check{\Lambda} \check{W}_{n}-\frac{1}{2}\left\{\check{W}_{n}^{2}, \check{\Lambda}\right\}+\mathcal{O}\left(W^{3}\right) .
\end{aligned}
$$

It follows from the normalization condition (1) that only 8 out of 16 matrix elements of $\breve{W}$ are independent parameters. This observation provides certain freedom to choose an explicit form of this matrix. A convenient parameterization to be used below is

$$
\check{W}_{n}=\left(\begin{array}{cccc}
0 & u_{1 n} & b_{1 n} & 0 \\
u_{2 n} & 0 & 0 & b_{2 n} \\
a_{1 n}+b_{1 n} & 0 & 0 & v_{1 n} \\
0 & a_{2 n}+b_{2 n} & v_{2 n} & 0
\end{array}\right) .
$$

With this choice the quadratic part of the action takes the form

$$
i S^{(2)}=i S_{a b}^{(2)}[a, b]+i S_{u v}^{(2)}[u, v],
$$

where $i S_{a b}^{(2)}[a, b]$ does not depend on $H$ and describes diffuson modes, while $i S_{u v}^{(2)}[u, v]$ is sensitive to the magnetic field and is responsible for the Cooperons. The diffuson part of the action $i S_{a b}^{(2)}[a, b]$ was already analyzed before $\frac{19}{19}$ and will be omitted here. Below we will focus our attention on the Cooperon contribution which reads

$$
\begin{aligned}
i S_{u v}^{(2)}[u, v]= & \sum_{n=1}^{N-1} \frac{2 \pi}{\delta_{n}} \operatorname{Tr}\left[\frac{\partial}{\partial t}\left[u_{1 n}, u_{2 n}\right]-16 \alpha_{n} H^{2} u_{1} u_{2}\right] \\
& +\sum_{n=1}^{N-1} \frac{2 \pi}{\delta_{n}} \operatorname{Tr}\left[\frac{\partial}{\partial t}\left[v_{2 n}, v_{1 n}\right]-16 \alpha_{n} H^{2} v_{1} v_{2}\right] \\
& -\sum_{n=1}^{N} \frac{g_{n}}{2} \operatorname{Tr}\left[\left(u_{1 n}-u_{1, n-1}\right)\left(u_{2 n}-u_{2, n-1}\right)\right. \\
& \left.+\left(v_{1 n}-v_{1, n-1}\right)\left(v_{2 n}-v_{2, n-1}\right)\right],
\end{aligned}
$$

where $g_{n}=2 \sum_{k} T_{k}^{(n)}=2 \pi \hbar / e^{2} R_{n}$ is the dimensionless conductance of $n$-th barrier. With the aid of the action (13) we can derive the pair correlators of the fields $u_{1,2}$ and $v_{1,2}$ :

$$
\begin{array}{r}
\left\langle u_{1 n}\left(t_{1}, t_{2}\right) u_{2 m}\left(t^{\prime}, t^{\prime \prime}\right)\right\rangle=\left\langle v_{1 n}\left(t^{\prime}, t^{\prime \prime}\right) v_{2 m}\left(t_{1}, t_{2}\right)\right\rangle \\
=\frac{\delta_{m}}{2 \pi} \delta\left(t_{1}-t_{2}+t^{\prime}-t^{\prime \prime}\right) C_{n m}\left(t^{\prime \prime}-t_{1}\right),
\end{array}
$$

where we defined a discrete version of the Cooperon $C_{n m}(t)$ obeying the equation

$$
\begin{array}{r}
\left(\frac{\partial}{\partial t}+\frac{1}{\tau_{H n}}+\frac{1}{\tau_{\varphi n}}\right) C_{n m}+\frac{\delta_{n}}{4 \pi}\left[\left(g_{n}+g_{n+1}\right) C_{n m}\right. \\
\left.-g_{n} C_{n-1, m}-g_{n+1} C_{n+1, m}\right]=\delta_{n m} \delta(t) .
\end{array}
$$

This equation should be supplemented by the boundary condition $C_{n m}(t)=0$ which applies whenever one of the indices $n$ or $m$ belongs to the lead electrode. Here $\tau_{H n}=1 / 16 \alpha_{n} H^{2}$ is the electron dephasing time due to the magnetic field. In Eq. (15) we also introduced an additional electron decoherence time in $n$-th quantum dot $\tau_{\varphi n}$ which can remain finite in the presence of interactions. In this section we account for electron decoherence only phenomenologically by keeping the parameter $\tau_{\varphi n}$ in the equation for the Cooperon. Rigorous description of quantum decoherence by electron-electron interactions will be carried out in Sec. 3 .

\section{Weak localization corrections to conductance}

Let us now derive an expression for WL correction to the conductance in terms of the fluctuating fields $u$ and $v$. In what follows we will explicitly account for the discrete nature of our model and specify the WL correction for a single barrier in-between two adjacent quantum dots in the array.

We start, however, from the bulk limit, in which case the Kubo formula for the conductivity tensor $\sigma_{\alpha \beta}$ reads

$$
\begin{aligned}
& \sigma_{\alpha \beta}\left(\boldsymbol{r}, \boldsymbol{r}^{\prime}\right)=-i \int_{-\infty}^{t} d t^{\prime}\left(t-t^{\prime}\right) \\
& \times\left\langle j_{\beta}\left(t^{\prime}, \boldsymbol{r}^{\prime}\right) j_{\alpha}(t, \boldsymbol{r})-j_{\alpha}(t, \boldsymbol{r}) j_{\beta}\left(t^{\prime}, \boldsymbol{r}^{\prime}\right)\right\rangle .
\end{aligned}
$$

Following the standard procedure $\stackrel{1,2}{2}$, approximating the Fermi function as $-\partial f_{F}(E) / \partial E \approx \delta(E)$ (which effectively implies taking the low temperature limit) and using a phenomenological description of interactions as mediated by external (classical) fluctuating fields $\underline{29}$, from Eq. (16) one can derive the WL correction in the form:

$$
\begin{aligned}
& \delta \sigma_{\alpha \beta}^{W L}\left(\boldsymbol{r}, \boldsymbol{r}^{\prime}\right)=-\frac{e^{2}}{4 \pi m^{2}} \int_{-\infty}^{t} d t^{\prime} \int d t^{\prime \prime} \\
& \times\left(\nabla_{\boldsymbol{r}_{1}}^{\alpha}-\nabla_{\boldsymbol{r}_{2}}^{\alpha}\right)_{\boldsymbol{r}_{1}=\boldsymbol{r}_{2}=\boldsymbol{r}}\left(\nabla_{\boldsymbol{r}_{1}^{\prime}}^{\beta}-\nabla_{\boldsymbol{r}_{2}^{\prime}}^{\beta}\right)_{\boldsymbol{r}_{1}^{\prime}=\boldsymbol{r}_{2}^{\prime}=\boldsymbol{r}^{\prime}} \\
& \times\left\langle G^{R}\left(t, \boldsymbol{r}_{1} ; t^{\prime \prime}, \boldsymbol{r}_{2}^{\prime}\right) G^{A}\left(t^{\prime}, \boldsymbol{r}_{1}^{\prime} ; t, \boldsymbol{r}_{2}\right)\right\rangle_{\mathrm{dis}, \max \text { cross }}
\end{aligned}
$$

which implies summation over all maximally crossed diagrams, as indicated in the subscript. At the same time, averaging over fluctuations of $\check{Q}$ within Gaussian approximation is equivalent to summing over all ladder diagrams. Since we are not going to go beyond the above approximation, we need to convert maximally crossed diagrams in Eq. (17) into the ladder ones. Technically this conversion can be accomplished by an effective time reversal procedure for the advanced Green function which can be illustrated as follows.

Consider, e. g., the second order correction to $G^{A}$ in the disorder potential $U_{\mathrm{dis}}(\boldsymbol{x})$

$$
\begin{aligned}
& { }^{(2)} G^{A}\left(t^{\prime}, \boldsymbol{r}_{1}^{\prime} ; t, \boldsymbol{r}_{2}\right)=-i \int_{t^{\prime}}^{t} d \tau_{2} \int_{t^{\prime}}^{\tau_{2}} d \tau_{1} \int d^{3} \boldsymbol{x}_{\mathbf{2}} d^{3} \boldsymbol{x}_{\mathbf{1}} \\
& \times G^{A}\left(t^{\prime}, \boldsymbol{r}_{1}^{\prime} ; \tau_{1}, \boldsymbol{x}_{1}\right) U_{\mathrm{dis}}\left(\boldsymbol{x}_{1}\right) G^{A}\left(\tau_{1}, \boldsymbol{x}_{1} ; \tau_{2}, \boldsymbol{x}_{2}\right) \\
& \times U_{\mathrm{dis}}\left(\boldsymbol{x}_{2}\right) G^{A}\left(\tau_{2}, \boldsymbol{x}_{2} ; t, \boldsymbol{r}_{2}\right) .
\end{aligned}
$$


Making use of the property $G^{A}\left(X_{1}, X_{2}\right)=G^{R *}\left(X_{2}, X_{1}\right)$, we get

$$
\begin{aligned}
& \delta^{(2)} G^{A}\left(t^{\prime}, \boldsymbol{r}_{1}^{\prime} ; t, \boldsymbol{r}_{2}\right)=-i \int_{t^{\prime}}^{t} d \tau_{2} \int_{t^{\prime}}^{\tau_{2}} d \tau_{1} \int d^{3} \boldsymbol{x}_{\mathbf{2}} d^{3} \boldsymbol{x}_{\mathbf{1}} \\
& \times G^{R *}\left(t, \boldsymbol{r}_{2} ; \tau_{2}, \boldsymbol{x}_{2}\right) U_{\mathrm{dis}}\left(\boldsymbol{x}_{2}\right) G^{R *}\left(\tau_{2}, \boldsymbol{x}_{2} ; \tau_{1}, \boldsymbol{x}_{1}\right) \\
& \times U_{\mathrm{dis}}\left(\boldsymbol{x}_{1}\right) G^{R *}\left(\tau_{1}, \boldsymbol{x}_{1} ; t^{\prime}, \boldsymbol{r}_{1}^{\prime}\right) .
\end{aligned}
$$

Setting $t_{f}=t+t^{\prime}$, we rewrite this expression as follows

$$
\begin{aligned}
& \delta^{(2)} G^{A}\left(t^{\prime}, \boldsymbol{r}_{1}^{\prime} ; t, \boldsymbol{r}_{2}\right)=-i \int_{t_{f}-t}^{t_{f}-t^{\prime}} d \tau_{2} \int_{t_{f}-t}^{\tau_{2}} d \tau_{1} \\
& \times \int d^{3} \boldsymbol{x}_{\mathbf{2}} d^{3} \boldsymbol{x}_{\mathbf{1}} G^{R *}\left(t_{f}-t^{\prime}, \boldsymbol{r}_{2} ; \tau_{2}, \boldsymbol{x}_{2}\right) \\
& \times U_{\mathrm{dis}}\left(\boldsymbol{x}_{2}\right) G^{R *}\left(\tau_{2}, \boldsymbol{x}_{2} ; \tau_{1}, \boldsymbol{x}_{1}\right) \\
& \times U_{\mathrm{dis}}\left(\boldsymbol{x}_{1}\right) G^{R *}\left(\tau_{1}, \boldsymbol{x}_{1} ; t_{f}-t, \boldsymbol{r}_{1}^{\prime}\right) .
\end{aligned}
$$

Close inspection of the right hand side of Eq. (20) allows to establish the following relation

$$
\delta^{(2)} G^{A}\left(t^{\prime}, \boldsymbol{r}_{1}^{\prime} ; t, \boldsymbol{r}_{2}\right)=\mathcal{T} \delta^{(2)} G^{R *}\left(t^{\prime}, \boldsymbol{r}_{2} ; t, \boldsymbol{r}_{1}^{\prime}\right) \mathcal{T},
$$

which turns out to hold in all orders of the perturbation theory in $U_{\text {dis }}$. As before, the time inversion operator $\mathcal{T}$ is defined in Eq. (8) with $t_{f}=t+t^{\prime}$.

As a result, the expression for $\delta \sigma_{\alpha \beta}^{W L}$ takes the form:

$$
\begin{aligned}
& \delta \sigma_{\alpha \beta}^{W L}\left(\boldsymbol{r}, \boldsymbol{r}^{\prime}\right)=-\frac{e^{2}}{4 \pi m^{2}} \int_{-\infty}^{t} d t^{\prime} \int d t^{\prime \prime} \\
& \times\left(\nabla_{\boldsymbol{r}_{1}}^{\alpha}-\nabla_{\boldsymbol{r}_{2}}^{\alpha}\right)_{\boldsymbol{r}_{1}=\boldsymbol{r}_{2}=\boldsymbol{r}}\left(\nabla_{\boldsymbol{r}_{1}^{\prime}}^{\beta}-\nabla_{\boldsymbol{r}_{2}^{\prime}}^{\beta}\right)_{\boldsymbol{r}_{1}^{\prime}=\boldsymbol{r}_{2}^{\prime}=\boldsymbol{r}^{\prime}} \\
& \times\left\langle G^{R}\left(t, \boldsymbol{r}_{1} ; t^{\prime \prime}, \boldsymbol{r}_{2}^{\prime}\right) \mathcal{T} G^{R *}\left(t^{\prime}, \boldsymbol{r}_{2} ; t, \boldsymbol{r}_{1}^{\prime}\right) \mathcal{T}\right\rangle_{\text {dis, ladder }}
\end{aligned}
$$

Rewriting Eq. (22) in terms of the matrix elements of the Green function (7), we obtain

$$
\begin{aligned}
& \delta \sigma_{\alpha \beta}^{W L}\left(\boldsymbol{r}, \boldsymbol{r}^{\prime}\right)=-\frac{e^{2}}{4 \pi m^{2}} \int_{-\infty}^{t} d t^{\prime} \int d t^{\prime \prime} \\
& \times\left(\nabla_{\boldsymbol{r}_{1}}^{\alpha}-\nabla_{\boldsymbol{r}_{2}}^{\alpha}\right)_{\boldsymbol{r}_{1}=\boldsymbol{r}_{2}=\boldsymbol{r}}\left(\nabla_{\boldsymbol{r}_{1}^{\prime}}^{\beta}-\nabla_{\boldsymbol{r}_{2}^{\prime}}^{\beta}\right)_{\boldsymbol{r}_{1}^{\prime}=\boldsymbol{r}_{2}^{\prime}=\boldsymbol{r}^{\prime}} \\
& \times\left\langle G_{33}\left(t, \boldsymbol{r}_{1} ; t^{\prime \prime}, \boldsymbol{r}_{2}^{\prime}\right) G_{44}\left(t^{\prime}, \boldsymbol{r}_{2} ; t, \boldsymbol{r}_{1}^{\prime}\right)\right\rangle_{\mathrm{dis}, \text { ladder }}
\end{aligned}
$$

Our next step amounts to expressing WL correction via the Green function $\breve{G}_{Q}$ (9). For that purpose we will use the following rule of averaging

$$
\begin{aligned}
& \left\langle G_{33}\left(t, \boldsymbol{r}_{1} ; t^{\prime \prime}, \boldsymbol{r}_{2}^{\prime}\right) G_{44}\left(t^{\prime}, \boldsymbol{r}_{2} ; t, \boldsymbol{r}_{1}^{\prime}\right)\right\rangle_{\mathrm{dis}} \\
& =\left\langle G_{33 ; Q}\left(t, \boldsymbol{r}_{1} ; t^{\prime \prime}, \boldsymbol{r}_{2}^{\prime}\right) G_{44 ; Q}\left(t^{\prime}, \boldsymbol{r}_{2} ; t, \boldsymbol{r}_{1}^{\prime}\right)\right\rangle_{Q} \\
& -\left\langle G_{34 ; Q}\left(t, \boldsymbol{r}_{1} ; t, \boldsymbol{r}_{1}^{\prime}\right) G_{43 ; Q}\left(t^{\prime}, \boldsymbol{r}_{2} ; t^{\prime \prime}, \boldsymbol{r}_{2}^{\prime}\right)\right\rangle_{Q} .
\end{aligned}
$$

One can check that within our Gaussian approximation in $u$ and $v$ the first term in the right hand side of Eq. (24) does not give any contribution. Hence, we find

$$
\begin{aligned}
& \delta \sigma_{\alpha \beta}^{W L}\left(\boldsymbol{r}, \boldsymbol{r}^{\prime}\right)=\frac{e^{2}}{4 \pi m^{2}} \int_{-\infty}^{t} d t^{\prime} \int d t^{\prime \prime} \\
& \times\left(\nabla_{\boldsymbol{r}_{1}}^{\alpha}-\nabla_{\boldsymbol{r}_{2}}^{\alpha}\right)_{\boldsymbol{r}_{1}=\boldsymbol{r}_{2}=\boldsymbol{r}}\left(\nabla_{\boldsymbol{r}_{1}^{\prime}}^{\beta}-\nabla_{\boldsymbol{r}_{2}^{\prime}}^{\beta}\right)_{\boldsymbol{r}_{1}^{\prime}=\boldsymbol{r}_{2}^{\prime}=\boldsymbol{r}^{\prime}} \\
& \times\left\langle G_{34 ; Q}\left(t, \boldsymbol{r}_{1} ; t, \boldsymbol{r}_{1}^{\prime}\right) G_{43 ; Q}\left(t^{\prime}, \boldsymbol{r}_{2} ; t^{\prime \prime}, \boldsymbol{r}_{2}^{\prime}\right)\right\rangle_{Q} .
\end{aligned}
$$

Let us now turn to our model of Fig. 1 in which case the voltage drops occur only across barriers. In this case Eq. (25), which only applies to bulk metals, should be generalized accordingly. Consider the conductance of an individual barrier determined by the following Kubo formula

$$
\begin{aligned}
G= & -i \int_{-\infty}^{t} d t^{\prime}\left(t-t^{\prime}\right)\left\langle I\left(t^{\prime}, x^{\prime}\right) I(t, x)\right. \\
& \left.-I(t, x) I\left(t^{\prime}, x^{\prime}\right)\right\rangle .
\end{aligned}
$$

Here $I(t, x)$ is the operator of the total current flowing in the lead (or dot) and $x$ is a longitudinal coordinate chosen to be in a close vicinity of the barrier. Due to the current conservation the conductance $G$ should not explicitly depend on $x$ and $x^{\prime}$. Comparing Eqs. (26) and (16), and making use of Eq. (25) and the relation $I(t, x)=\int d^{2} \boldsymbol{z} j_{x}(t, x, \boldsymbol{z})$, where $j_{x}$ is the current density in the $x$-direction and $z$ is the vector in the transversal direction, we conclude that WL correction to the conductance of a barrier between the left and right dots should read

$$
\begin{aligned}
& \delta G_{L R}^{W L}=\frac{e^{2}}{4 \pi m^{2}} \int_{-\infty}^{t} d t^{\prime} \int d t^{\prime \prime} \int d^{2} \boldsymbol{z} d^{2} \boldsymbol{z}^{\prime} \\
& \times\left(\nabla_{x_{1}}-\nabla_{x_{2}}\right)_{x_{1}=x_{2}=x}\left(\nabla_{x_{1}^{\prime}}-\nabla_{x_{2}^{\prime}}\right)_{x_{1}^{\prime}=x_{2}^{\prime}=x^{\prime}} \\
& \times\left\langle G_{34 ; Q}\left(t, x_{1}, \boldsymbol{z} ; t, x_{1}^{\prime}, \boldsymbol{z}^{\prime}\right) G_{43 ; Q}\left(t^{\prime}, x_{2}, \boldsymbol{z} ; t^{\prime \prime}, x_{2}^{\prime}, \boldsymbol{z}^{\prime}\right)\right\rangle_{Q} .
\end{aligned}
$$

In what follows we will assume that both coordinates $x$ and $x^{\prime}$ are on the left side from and very close to the corresponding barrier. Let us express the Green function in the vicinity of the barrier in the form

$$
\begin{aligned}
& \check{G}_{Q}\left(t, x, \boldsymbol{z} ; t^{\prime}, x^{\prime}, \boldsymbol{z}^{\prime}\right)=\sum_{n m}\left\{e^{i p_{n} x_{1}-i p_{m} x^{\prime}} \check{\mathcal{G}}_{m n}^{++}\left(t, t^{\prime}, x, x^{\prime}\right)\right. \\
& +e^{-i p_{n} x+i p_{m} x^{\prime}} \check{\mathcal{G}}_{m n}^{--}\left(t, t^{\prime}, x, x^{\prime}\right) \\
& +e^{i p_{n} x+i p_{m} x^{\prime}} \check{\mathcal{G}}_{m n}^{+-}\left(t, t^{\prime}, x, x^{\prime}\right) \\
& \left.+e^{-i p_{n} x-i p_{m} x^{\prime}} \check{\mathcal{G}}_{m n}^{-+}\left(t, t^{\prime}, x, x^{\prime}\right)\right\} \Phi_{n}(\boldsymbol{z}) \Phi_{m}^{*}\left(\boldsymbol{z}^{\prime}\right),
\end{aligned}
$$

where $\Phi_{n}(\boldsymbol{z})$ are the transverse quantization modes which define conducting channels, $p_{n}$ is projection of the Fermi momentum perpendicular to the surface of the barrier, and the semiclassical Green function $\mathcal{G}_{m n}^{\alpha \beta}$ slowly varies in space. Eq. (27) then becomes

$$
\begin{aligned}
& \delta G_{L R}^{W L}=\frac{e^{2}}{4 \pi m^{2}} \int_{-\infty}^{t} d t^{\prime} \int d t^{\prime \prime} \\
& \times \sum_{m n k l} \sum_{\alpha \beta \gamma \delta= \pm 1}\left(\alpha p_{n}-\gamma p_{k}\right)\left(\beta p_{m}-\delta p_{l}\right) \\
& \times\left\langle\mathcal{G}_{m n ; 34}^{\alpha \beta}\left(t, t, x, x^{\prime}\right) \mathcal{G}_{k l ; 43}^{\gamma \delta}\left(t^{\prime}, t^{\prime \prime}, x, x^{\prime}\right)\right\rangle_{Q} \\
& \times\left. e^{i \alpha p_{n} x_{1}-i \beta p_{m} x_{1}^{\prime}+i \gamma p_{k} x_{2}-i \delta p_{l} x_{2}^{\prime}}\right|_{x_{1}=x_{2}=x ; x_{1}^{\prime}=x_{2}^{\prime}=x^{\prime}} .
\end{aligned}
$$

Next we require $\delta G_{L R}^{W L}$ to be independent on $x$ and $x^{\prime}$, i.e. in Eq. (29) we omit those terms, which contain 
quickly oscillating functions of these coordinates. This requirement implies that $\alpha p_{n}+\gamma p_{k}=0$ and $\beta p_{m}+\delta p_{l}=$ 0 . These constraints in turn yield $\gamma=-\alpha, \delta=-\beta$, $k=n$ and $l=m$. Thus, we get

$$
\begin{gathered}
\delta G_{L R}^{W L}=\frac{e^{2}}{\pi m^{2}} \sum_{m n} \sum_{\alpha \beta= \pm 1} \int_{-\infty}^{t} d t^{\prime} \int d t^{\prime \prime} \alpha \beta p_{n} p_{m} \\
\times\left\langle\mathcal{G}_{m n ; 34}^{\alpha \beta}\left(t, t, x, x^{\prime}\right) \mathcal{G}_{n m ; 43}^{-\alpha,-\beta}\left(t^{\prime}, t^{\prime \prime}, x, x^{\prime}\right)\right\rangle_{Q} .
\end{gathered}
$$

Let us choose the basis in which transmission and reflection matrices $\hat{t}$ and $\hat{r}$ are diagonal. In this basis the semiclassical Green function is diagonal as well, $\mathcal{G}_{m n} \propto \mathcal{G}_{n n} \delta_{n m}$, and Eq. (30) takes the form

$$
\begin{aligned}
\delta G_{L R}^{W L}= & \frac{e^{2}}{\pi} \sum_{n} \frac{p_{n}^{2}}{m^{2}} \int_{-\infty}^{t} d t^{\prime} \int d t^{\prime \prime} \\
& \times\left\langle\mathcal{G}_{L, n n ; 34}^{++}(t, t) \mathcal{G}_{L, n n ; 43}^{--}\left(t^{\prime}, t^{\prime \prime}\right)\right. \\
& +\mathcal{G}_{L, n n ; 34}^{--}(t, t) \mathcal{G}_{L, n n ; 43}^{++}\left(t^{\prime}, t^{\prime \prime}\right) \\
& -\mathcal{G}_{L, n n ; 34}^{+-}(t, t) \mathcal{G}_{L, n n ; 43}^{-+}\left(t^{\prime}, t^{\prime \prime}\right) \\
& \left.-\mathcal{G}_{L, n n ; 34}^{-+}(t, t) \mathcal{G}_{L, n n ; 43}^{+-}\left(t^{\prime}, t^{\prime \prime}\right)\right\rangle_{Q} .
\end{aligned}
$$

What remains is to express the WL correction in terms of the field $\check{Q}$ only. This goal is achieved with the aid of the following general relation 23

$$
\begin{aligned}
& \delta G_{L R}^{W L}=-\frac{e^{2}}{\pi} \sum_{n} \int_{-\infty}^{t} d t^{\prime} \int d t^{\prime \prime} \\
& \times\left\langle T_{n}\left[v_{1 L}(t, t) v_{2 R}\left(t^{\prime}, t^{\prime \prime}\right)+v_{1 R}(t, t) v_{2 L}\left(t^{\prime}, t^{\prime \prime}\right)\right]\right. \\
& \left.+T_{n}^{2}\left[v_{1 L}(t, t)-v_{1 R}(t, t)\right]\left[v_{2 L}\left(t^{\prime}, t^{\prime \prime}\right)-v_{2 R}\left(t^{\prime}, t^{\prime \prime}\right)\right]\right\rangle .
\end{aligned}
$$

Note that the contribution linear in $T_{n}$, which contains the product of the fluctuating fields on two different sides of the barrier, vanishes identically provided fluctuations on one side tend to zero, e.g. if the barrier is directly attached to a large metallic lead. In contrast, the contribution $\propto T_{n}^{2}$ in Eq. (32) survives even in this case.

Finally, applying the contraction rule (14) we get

$$
\begin{aligned}
\delta G_{L R}^{W L}= & -\frac{e^{2} g}{4 \pi^{2}} \int_{0}^{\infty} d t\left\{\beta\left[\delta_{R} C_{L R}(t)+\delta_{L} C_{R L}(t)\right]\right. \\
& \left.+(1-\beta)\left[\delta_{R} C_{R R}(t)+\delta_{L} C_{L L}(t)\right]\right\}
\end{aligned}
$$

Here $\delta_{L, R}$ is the mean level spacing in the left/right quantum dot,

$$
g=2 \sum_{k} T_{k}
$$

is the dimensionless conductance of the barrier and

$$
\beta=\sum_{k} T_{k}\left(1-T_{k}\right) / \sum_{k} T_{k}
$$

is the corresponding Fano factor.

Likewise, the WL correction to the $n$-th barrier conductance in 1d array of $N-1$ quantum dots with mean

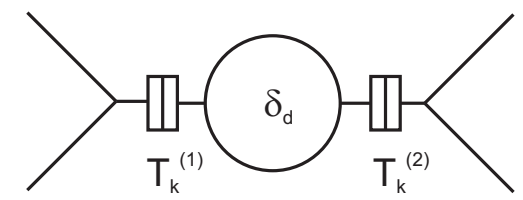

FIG. 2: Single quantum dot connected to the leads via two barriers.

level spacings $\delta_{n}$ connected by $N$ barriers with dimensionless conductances $g_{n}$ and Fano factors $\beta_{n}$ reads

$$
\begin{aligned}
\delta G_{n}^{W L}= & -\frac{e^{2} g_{n}}{4 \pi^{2}} \int_{0}^{\infty} d t\left\{\beta _ { n } \left[\delta_{n} C_{n-1, n}(t)\right.\right. \\
& \left.+\delta_{n-1} C_{n, n-1}(t)\right]+\left(1-\beta_{n}\right)\left[\delta_{n} C_{n n}(t)\right. \\
& \left.\left.+\delta_{n-1} C_{n-1, n-1}(t)\right]\right\} .
\end{aligned}
$$

So far we discussed the local properties, namely WL corrections to the conductivity tensor, $\delta \sigma_{\alpha, \beta}^{W L}\left(\boldsymbol{r}, \boldsymbol{r}^{\prime}\right)$, and to the conductance of a single barrier, $\delta G_{L R}^{W L}$. Our main goal is, however, to evaluate the WL correction to the conductance of the whole system. For bulk metals one finds that at large scales the WL correction (17) is local, $\delta \sigma_{\alpha, \beta}^{W L}\left(\boldsymbol{r}, \boldsymbol{r}^{\prime}\right) \propto \delta\left(\boldsymbol{r}-\boldsymbol{r}^{\prime}\right)$. In general though, there can exist other, non-local, contributions to the conductivity tensor ${ }^{30}$. Without going into details here, we only point out that, even if these non-local terms are present, one can still apply the standard Ohm's law arguments in order to obtain the conductance of the whole sample. Specifically, in the case of $1 \mathrm{~d}$ arrays one finds ${ }^{23}$ (see also 31 )

$$
\begin{aligned}
\delta G^{W L} & =\frac{1}{\sum_{n=1}^{N}\left(G_{n}+\delta G_{n}^{W L}\right)^{-1}}-\frac{1}{\sum_{n=1}^{N} G_{n}^{-1}} \\
& =\frac{\sum_{n=1}^{N} \delta G_{n}^{W L} / g_{n}^{2}}{\left(\sum_{n=1}^{N} 1 / g_{n}\right)^{2}}+\text { higher order terms. }
\end{aligned}
$$

Eqs. (33), (36) and (37) will be used to evaluate WL corrections for different configurations of quantum dots considered below.

\section{Examples}

\section{Single quantum dot}

We start from the simplest case of a single quantum dot depicted in Fig. 2. In this case the solution of Eq. (15) reads

$$
C_{11}(t)=\exp \left[-\frac{t}{\tau_{D}}-\frac{t}{\tau_{H}}-\frac{t}{\tau_{\varphi}}\right]
$$

where $\tau_{D}=4 \pi /\left(g_{1}+g_{2}\right) \delta_{d}$ is the dwell time, and $\delta_{d}$ is the mean level spacing in the quantum dot. All other 


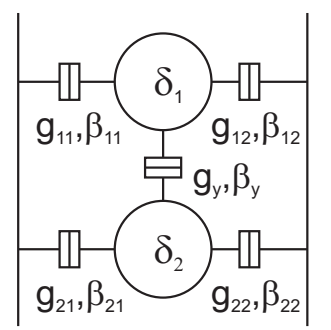

FIG. 3: Most general system with two quantum dots

components of the Cooperon are equal to zero. From Eq. (33) we get

$$
\begin{aligned}
\delta G_{1}^{W L} & =-\frac{e^{2} g_{1}\left(1-\beta_{1}\right) \delta_{d}}{4 \pi^{2}} \frac{1}{1 / \tau_{D}+1 / \tau_{H}+1 / \tau_{\varphi}} \\
\delta G_{2}^{W L} & =-\frac{e^{2} g_{2}\left(1-\beta_{2}\right) \delta_{d}}{4 \pi^{2}} \frac{1}{1 / \tau_{D}+1 / \tau_{H}+1 / \tau_{\varphi}} .
\end{aligned}
$$

According to Eq. (37) the total WL correction becomes

$$
\delta G^{W L}=-\frac{e^{2} \delta}{4 \pi^{2}} \frac{g_{1} g_{2}^{2}\left(1-\beta_{1}\right)+g_{1}^{2} g_{2}\left(1-\beta_{2}\right)}{\left(g_{1}+g_{2}\right)^{2}\left(1 / \tau_{D}+1 / \tau_{\varphi}+1 / \tau_{H}\right)} .
$$

Since $1 / \tau_{H} \propto H^{2}$, the magnetoconductance has the Lorentzian shape $\frac{13}{2}$. In the limit $H=0$ and in the absence of interactions $\left(\tau_{\varphi} \rightarrow \infty\right)$ Eq. (40) reduces to ${ }^{32}$

$$
\delta G^{W L}=-\frac{e^{2}}{\pi} \frac{g_{1} g_{2}^{2}\left(1-\beta_{1}\right)+g_{1}^{2} g_{2}\left(1-\beta_{2}\right)}{\left(g_{1}+g_{2}\right)^{3}} .
$$

As one can see for the case of low transmissions (for example in case of tunneling barriers) the WL corrections equals to zero.

\section{Two quantum dots}

Next we consider the most general setup composed of two quantum dots with the corresponding conductances and Fano factors defined as in Fig. 3. The Cooperon is represented as a $2 \times 2$ matrix which zero frequency component satisfies the following equation

$$
\begin{array}{r}
\left(\begin{array}{cc}
g_{11}+g_{12}+g_{y}+\gamma_{1} & -g_{y} \\
-g_{y} & g_{21}+g_{22}+g_{y}+\gamma_{2}
\end{array}\right)\left(\begin{array}{ll}
C_{11} & C_{12} \\
C_{21} & C_{22}
\end{array}\right) \\
=\left(\begin{array}{cc}
4 \pi / \delta_{1} & 0 \\
0 & 4 \pi / \delta_{2}
\end{array}\right)
\end{array}
$$

where

$$
\gamma_{1,2}=\frac{4 \pi}{\delta_{1,2}}\left(\frac{1}{\tau_{H 1,2}}+\frac{1}{\tau_{\varphi 1,2}}\right) .
$$

Defining $\Delta=\left(g_{11}+g_{12}+g_{y}+\gamma_{1}\right)\left(g_{21}+g_{22}+g_{y}+\gamma_{2}\right)-g_{y}^{2}$, we get

$$
\left(\begin{array}{ll}
C_{11} & C_{12} \\
C_{21} & C_{22}
\end{array}\right)=\frac{4 \pi}{\Delta}\left(\begin{array}{cc}
\left(g_{21}+g_{22}+g_{y}+\gamma_{2}\right) / \delta_{1} & g_{y} / \delta_{2} \\
g_{y} / \delta_{1} & \left(g_{11}+g_{12}+g_{y}+\gamma_{1}\right) / \delta_{2}
\end{array}\right) .
$$

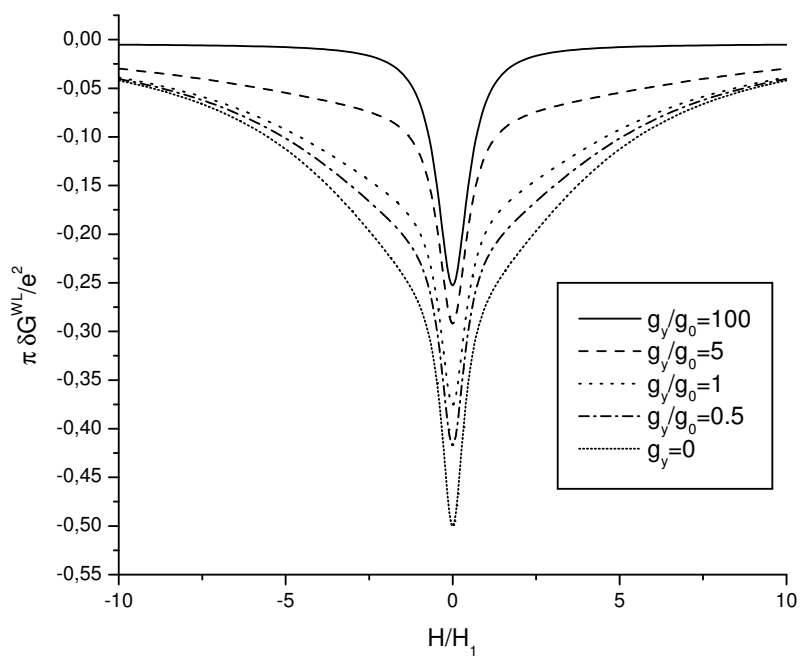

FIG. 4: The magnetoconductance of two dots of Fig. 3 for $d_{1}, d_{2} \gg l_{e}, d_{1} / d_{2}=5, g_{i j}=g_{0}, \beta_{i j}=0, \beta_{y}=0, \tau_{\varphi 1}=$ $\tau_{\varphi 2}=\infty$. Here $H_{1}=1 / 4 \sqrt{\alpha_{1} \tau_{D 1}}$ is the field at which weak localization is effectively suppressed in the first dot. For $g_{y}=$ 0 the magnetoconductance is given by superposition of two Lorentzians with different widths (decoupled dots), while for large $g_{y}$ only one Lorentzian survives corresponding to the contribution of a one "composite dot".

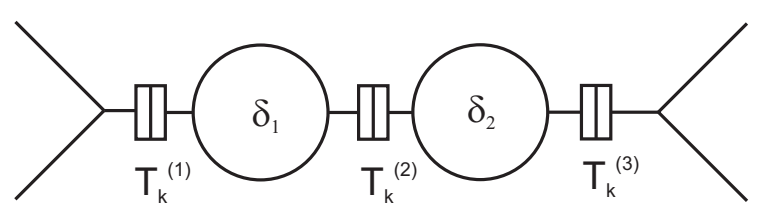

FIG. 5: Two quantum dots in series.

With the aid of Eq. (33) we can derive WL corrections for all five barriers in our setup which we do not specify here for the sake of brevity (see $\frac{23}{3}$ for further details).

WL correction to the conductance of the whole structure $\delta G^{W L}$ is obtained from the general expression for the conductance determined by Ohm's law:

$$
\begin{aligned}
G= & {\left[G_{11} G_{12}\left(G_{21}+G_{22}\right)+G_{21} G_{22}\left(G_{11}+G_{12}\right)\right.} \\
& \left.+G_{y}\left(G_{12}+G_{22}\right)\left(G_{11}+G_{21}\right)\right] \\
& /\left[\left(G_{11}+G_{12}\right)\left(G_{21}+G_{22}\right)\right. \\
& \left.+G_{y}\left(G_{11}+G_{12}+G_{21}+G_{22}\right)\right]
\end{aligned}
$$

Substituting $G_{i j} \rightarrow G_{i j}+\delta G_{i j}^{W L}$ into this formula and expanding the result to the first order in $\delta G_{i j}^{W L}$, we get

$$
\delta G^{W L}=\sum_{i, j=1,2} \frac{\partial G}{\partial G_{i j}} \delta G_{i j}^{W L}+\frac{\partial G}{\partial G_{y}} \delta G_{y}^{W L} .
$$

This general result for the WL correction to the conductance is illustrated in Fig. 4for a particular choice of the system parameters.

Of particular importance for us here is the system of two quantum dots connected in series, as shown in Fig. 5 , 
i.e. in the general structure of Fig. 3 we set $G_{12}=G_{21}=$ $0, G_{11}=G_{1}, G_{y}=G_{2}, G_{22}=G_{3}, \beta_{11}=\beta_{1}, \beta_{y}=\beta_{2}$ and $\beta_{22}=\beta_{3}$. We also assume $H=0$ and $\tau_{\varphi}=\infty$. WL corrections to the barrier conductances then take the form

$$
\begin{aligned}
\delta G_{1}^{W L} & =-\frac{e^{2}}{\pi} \frac{g_{1}\left(g_{2}+g_{3}\right)\left(1-\beta_{1}\right)}{g_{1} g_{2}+g_{2} g_{3}+g_{1} g_{3}} \\
\delta G_{2}^{W L} & =-\frac{e^{2}}{\pi} \frac{g_{2}\left(g_{1}+g_{3}\right)\left(1-\beta_{2}\right)+2 g_{2}^{2}}{g_{1} g_{2}+g_{2} g_{3}+g_{1} g_{3}} \\
\delta G_{3}^{W L} & =-\frac{e^{2}}{\pi} \frac{g_{3}\left(g_{1}+g_{2}\right)\left(1-\beta_{3}\right)}{g_{1} g_{2}+g_{2} g_{3}+g_{1} g_{3}}
\end{aligned}
$$

while Eq. (44) reduces to

$$
G=\frac{G_{1} G_{2} G_{3}}{G_{1} G_{2}+G_{1} G_{3}+G_{2} G_{3}} .
$$

WL correction for the whole system then reads

$$
\begin{aligned}
\delta G^{W L}= & -\frac{e^{2}}{\pi} \frac{g_{1} g_{2}^{2} g_{3}^{2}\left(g_{2}+g_{3}\right)\left(1-\beta_{1}\right)}{\left(g_{1} g_{2}+g_{2} g_{3}+g_{1} g_{3}\right)^{3}} \\
& -\frac{e^{2}}{\pi} \frac{g_{1}^{2} g_{2} g_{3}^{2}\left(g_{1}+g_{3}\right)\left(1-\beta_{2}\right)}{\left(g_{1} g_{2}+g_{2} g_{3}+g_{1} g_{3}\right)^{3}} \\
& -\frac{e^{2}}{\pi} \frac{g_{1}^{2} g_{2}^{2} g_{3}\left(g_{1}+g_{2}\right)\left(1-\beta_{3}\right)}{\left(g_{1} g_{2}+g_{2} g_{3}+g_{1} g_{3}\right)^{3}} \\
& -\frac{2 e^{2}}{\pi} \frac{g_{1}^{2} g_{2}^{2} g_{3}^{2}}{\left(g_{1} g_{2}+g_{2} g_{3}+g_{1} g_{3}\right)^{3}} .
\end{aligned}
$$

In the limit of open quantum dots, i.e. $\beta_{1,2,3}=0$, we reproduce the result ${ }^{31}$. It is easy to see that provided the conductance of one of the barriers strongly exceeds two others, Eq. (48) reduces to Eq. (41). If all three barriers are tunnel junctions, $\beta_{1,2,3} \rightarrow 1$, the first three contributions in Eq. (48) vanish, and only the last contribution - independent of the Fano factors - survives in this limit. If, on top of that, one of the tunnel junctions, e.g. the central one, is less transparent than two others, $g_{2} \ll g_{1}, g_{3}$, the result acquires a particularly simple (non-Lorentzian) form

$$
\delta G^{W L}=-\frac{2 e^{2}}{\pi} \frac{g_{2}^{2}}{\left(g_{1}+\gamma_{1}\right)\left(g_{3}+\gamma_{2}\right)},
$$

with $\gamma_{1,2}$ defined in Eq. (43). Note that $\delta G^{W L} \propto g_{2}^{2}$, i.e. this result is dominated by the second order tunneling processes across the second barrier.

\section{1D array of identical quantum dots}

Let us now turn to 1d arrays of quantum dots depicted in Fig. 1. For simplicity, we will assume that our array consists of $N-1$ identical quantum dots with the same level spacing $\delta_{n} \equiv \delta_{d}$ and of $N$ identical barriers with the same dimensionless conductance $g_{n} \equiv g$ and the same Fano factor $\beta_{n} \equiv \beta$. We will also assume that the quantum dots have the same shape and size so that $\tau_{H n} \equiv \tau_{H}$ and $\tau_{\varphi n} \equiv \tau_{\varphi}$. For this system the Cooperon can also be found exactly. The result reads

$$
C_{n m}(\omega)=\frac{2}{N} \sum_{q=1}^{N-1} \frac{\sin \frac{\pi q n}{N} \sin \frac{\pi q m}{N}}{-i \omega+\frac{1}{\tau_{H}}+\frac{1}{\tau_{\varphi}}+\frac{1-\cos \frac{\pi q}{N}}{\tau_{D}}} .
$$

Here $\tau_{D}=2 \pi / g \delta_{d}$ and $\tau_{H}=1 / 16 \alpha H^{2}$. The WL correction then takes the form

$$
\delta G^{W L}=-\frac{e^{2} g \delta_{d}}{2 \pi^{2} N^{2}} \sum_{q=1}^{N-1} \frac{\beta \cos \frac{\pi q}{N}+1-\beta}{\frac{1}{\tau_{H}}+\frac{1}{\tau_{\varphi}}+\frac{1-\cos \frac{\pi q}{N}}{\tau_{D}}} .
$$

The sum over $q$ can be handled exactly and yields

$$
\begin{aligned}
& \delta G^{W L}=-\frac{e^{2}}{\pi N^{2}}\left[\left(N \frac{1+u^{2 N}}{1-u^{2 N}}-\frac{1+u^{2}}{1-u^{2}}\right)\right. \\
& \left.\times \frac{\beta\left(1+u^{2}\right)+2(1-\beta) u}{1-u^{2}}-(N-1) \beta\right],
\end{aligned}
$$

where

$$
u=1+\frac{\tau_{D}}{\tau_{H}}+\frac{\tau_{D}}{\tau_{\varphi}}-\sqrt{\left(1+\frac{\tau_{D}}{\tau_{H}}+\frac{\tau_{D}}{\tau_{\varphi}}\right)^{2}-1}
$$

In the tunneling limit $\beta=1$ and for $\tau_{\varphi} \rightarrow \infty$ our result defined in Eqs. (52)- (53) becomes similar - though not exactly identical - to the corresponding result ${ }^{33}$.

If $\tau_{\varphi}$ is long enough, namely $1 / \tau_{\varphi} \lesssim E_{\mathrm{Th}}$, where $E_{\mathrm{Th}}=$ $\pi^{2} / 2 N^{2} \tau_{D}$ is the Thouless energy of the whole array, in Eqs. (51)-(52) it is sufficient to set $\tau_{\varphi}=\infty$. In this case the magnetic field $H$ significantly suppresses $\mathrm{WL}$ correction provided $1 / \tau_{H} \gtrsim E_{\mathrm{Th}}$ or, equivalently, if

$$
H \gtrsim H_{N}, \quad H_{N}=\frac{1}{8 N} \sqrt{\frac{\pi g \delta_{d}}{\alpha}} .
$$

In the opposite limit $1 / \tau_{\varphi} \gtrsim E_{\mathrm{Th}}$ we find

$$
\delta G^{W L}=-\frac{e^{2}}{\pi N}\left[\frac{\beta\left(1+\frac{\tau_{D}}{\tau_{H}}+\frac{\tau_{D}}{\tau_{\varphi}}\right)+1-\beta}{\sqrt{\left(1+\frac{\tau_{D}}{\tau_{H}}+\frac{\tau_{D}}{\tau_{\varphi}}\right)^{2}-1}}-\beta\right] .
$$

In particular, in the diffusive limit $\tau_{H}, \tau_{\varphi} \gg \tau_{D}$ we get

$$
\delta G^{W L}=-\frac{e^{2}}{\pi N d} \sqrt{\frac{D \tau_{H} \tau_{\varphi}}{\tau_{H}+\tau_{\varphi}}}
$$

where we introduced the diffusion coefficient

$$
D=d^{2} / 2 \tau_{D}
$$

Eq. (56) coincides with the standard result for quasi1d diffusive metallic wire. Note, however, that the values of $\tau_{H}$ within our model may differ from those for a metallic wire. The ratio of the former to the latter is $\tau_{H}^{\mathrm{qd}} / \tau_{H}^{\mathrm{met}} \sim \tau_{\mathrm{fl}} / \tau_{D}$, where $\tau_{\mathrm{fl}} \sim d / v_{F}$ is the flight time through the quantum dot. Since typically $\tau_{\mathrm{fl}}<\tau_{D}$ we 


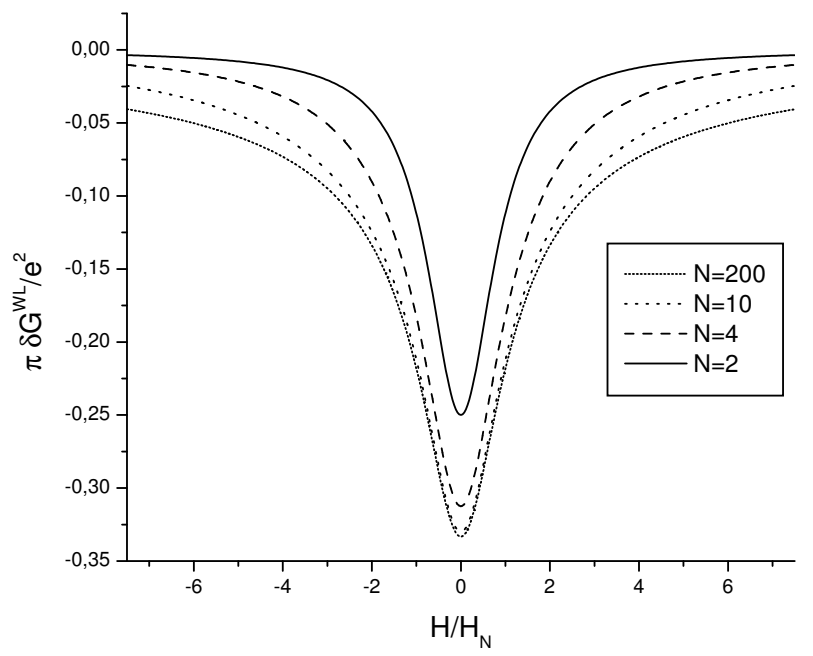

FIG. 6: Magnetoconductance of a $1 \mathrm{~d}$ array of $N-1$ identical open $(\beta=0)$ quantum dots in the absence of interactions $\left(\tau_{\varphi} \rightarrow \infty\right)$. The field $H_{N}$ is defined in Eq. (54).

conclude that for the same value of $D$ the magnetic field dephases electrons stronger in the case of an array of quantum dots.

For a single quantum dot $(N=2)$ Eq. (52) reduces to

$$
\delta G^{W L}=-\frac{e^{2}(1-\beta)}{4 \pi} \frac{1}{\left(1+\frac{\tau_{D}}{\tau_{H}}+\frac{\tau_{D}}{\tau_{\varphi}}\right)}
$$

in agreement with Eq. (40).

For two identical quantum dots in series we obtain

$$
\delta G^{W L}=-\frac{e^{2}}{9 \pi}\left[\frac{2-\beta}{1+\frac{2 \tau_{D}}{\tau_{H}}+\frac{2 \tau_{D}}{\tau_{\varphi}}}+\frac{\frac{2}{3}-\beta}{1+\frac{2 \tau_{D}}{3 \tau_{H}}+\frac{2 \tau_{D}}{3 \tau_{\varphi}}}\right],
$$

i.e. the magnetoconductance is just the sum of two Lorentzians in this case.

Finally, in the absence of any interactions $\left(\tau_{\varphi}=\infty\right)$ and at $H=0$ we obtain

$$
\delta G^{W L}=-\frac{e^{2}}{\pi}\left[\frac{1}{3}-\frac{\beta}{N}+\frac{1}{N^{2}}\left(\beta-\frac{1}{3}\right)\right] .
$$

In the limit $N \rightarrow \infty$ this result reduces to the standard one for a long quasi- $1 \mathrm{~d}$ diffusive wire ${ }^{34}$ while for any finite $N$ we reproduce the results for tunnel barriers $33(\beta \rightarrow 1)$ and open quantum $\operatorname{dots}^{35}(\beta \rightarrow 0)$.

The magnetoconductance of a $1 \mathrm{~d}$ array of $N-1$ identical open quantum dots in the absence of interactions is also illustrated in Fig. 6.

\section{QUANTUM DECOHERENCE BY ELECTRON-ELECTRON INTERACTIONS}

\section{A. Qualitative arguments}

Let us now include electron-electron interactions and analyze their impact on loss of phase coherence of elec-

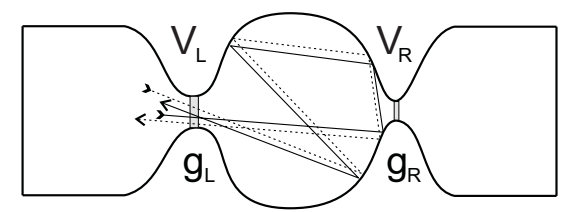

FIG. 7: Single quantum dot and a pair of time-reversed electron paths. Fluctuating voltages $V_{L}$ and $V_{R}$ are assumed to drop only across the barriers and not inside the dot.

trons' wave functions. Before turning to a detailed calculation it is instructive to discuss a simple qualitative picture demonstrating under which conditions decoherence by electron-electron interactions is expected to occur.

Consider first the simplest system of two scatterers separated by a cavity (quantum dot, Fig. 7) The WL correction to conductance of a disordered system $G_{W L}$ is known to arise from interference of pairs of time-reversed electron paths ${ }^{2}$. In the absence of interactions for a single quantum dot of Fig. 7 this correction was evaluated in the previous sections (see Eq. (41)). The effect of electron-electron interactions can be described in terms of fluctuating voltages. Let us assume that the voltage can drop only across the barriers and consider two timereversed electron paths which cross the left barrier (with fluctuating voltage $V_{L}(t)$ ) twice at times $t_{i}$ and $t_{f}$, as it is shown in Fig. 7. It is easy to see that the voltagedependent random phase factor $\exp \left(i \int_{t_{i}}^{t_{f}} V_{L}(t) d t\right)$ acquired by the electron wave function $\Psi$ along any path turns out to be exactly the same as that for its timereversed counterpart. Hence, in the product $\Psi \Psi^{*}$ these random phases cancel each other and quantum coherence of electrons remains fully preserved. This implies that for the system of Fig. 9 fluctuating voltages (which can mediate electron-electron interactions) do not cause any dephasing.

This qualitative conclusion can be verified by means of more rigorous considerations. For instance, it was demonstrated 18 that the scattering matrix of the system remains unitary in the presence of electron-electron interactions, which implies that the only effect of such interactions is transmission renormalization but not electron decoherence. A similar conclusion was reached ${ }^{36}$ by directly evaluating the WL correction to the system conductance. Thus, for the system of two scatterers of Fig. 7 electron-electron interactions can only yield energy dependent (logarithmic at sufficiently low energies) renormalization of the dot channel transmissions ${ }^{18,20}$ but not electron dephasing.

Let us now add one more scatterer and consider the system of two quantum dots depicted in Fig. 8. We again assume that fluctuating voltages are concentrated at the barriers and not inside the cavities. The phase factor accumulated along the path (see Fig. 8) which crosses the central barrier twice (at times $t_{i}$ and $t>t_{i}$ ) and returns to the initial point (at a time $t_{f}$ ) is $e^{i\left[\varphi\left(t_{i}\right)-\varphi(t)\right]}$, where $\dot{\varphi} / e=V(t)$ is the fluctuating voltage across the central 


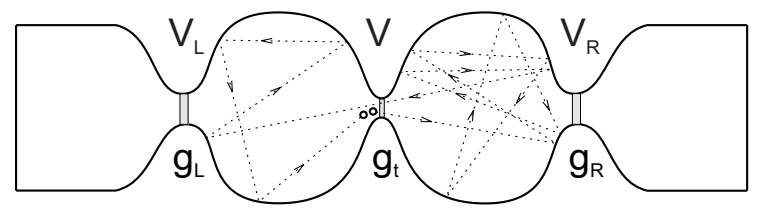

FIG. 8: Two quantum dots and a typical electron path. Fluctuating voltages $V_{L}, V$ and $V_{R}$ are again assumed to drop only across the barriers.

barrier. Similarly, the phase factor picked up along the time-reversed path reads $e^{i\left[\varphi\left(t_{f}+t_{i}-t\right)-\varphi\left(t_{f}\right)\right]}$. Hence, the overall phase factor acquired by the product $\Psi \Psi^{*}$ for a pair of time-reversed paths is $\exp \left(i \Phi_{\text {tot }}\right)$, where

$$
\Phi_{\mathrm{tot}}\left(t_{i}, t_{f}, t\right)=\varphi\left(t_{i}\right)-\varphi(t)-\varphi^{+}\left(t_{f}+t_{i}-t\right)+\varphi\left(t_{f}\right) .
$$

Averaging over phase fluctuations, which for simplicity are assumed Gaussian, we obtain

$$
\begin{aligned}
& \left\langle e^{i \Phi_{t o t}\left(t_{i}, t_{f}, t\right)}\right\rangle=e^{-\frac{1}{2}\left\langle\Phi_{t o t}^{2}\left(t_{i}, t_{f}, t\right)\right\rangle} \\
& =e^{-2 F\left(t-t_{i}\right)-2 F\left(t_{f}-t\right)+F\left(t_{f}-t_{i}\right)+F\left(t_{f}+t_{i}-2 t\right)},
\end{aligned}
$$

where we defined the phase correlation function

$$
F(t)=\left\langle(\varphi(t)-\varphi(0))^{2}\right\rangle / 2 .
$$

Should this function grow with time the electron phase coherence decays and, hence, $G_{W L}$ has to be suppressed below its non-interacting value due to interactioninduced electron decoherence.

The above arguments are, of course, not specific to systems with three barriers only. They can also be applied to any system with larger number of scatterers, i.e. virtually to any disordered conductor where - exactly for the same reasons - one also expects non-vanishing interactioninduced electron decoherence at any temperature includ$\operatorname{ing} T=0$. Below we will develop a quantitative theory which will confirm and extend our qualitative physical picture. We are going to give a complete quantum mechanical analysis of the problem which fully accounts for Fermi statistics of electrons and treats electron-electron interactions in terms quantum fields produced internally by fluctuating electrons.

\section{B. Nanorings with two quantum dots}

\section{The model and basic formalism}

Consider the system depicted in Fig. 9. The structure consists of two chaotic quantum dots (L and R) characterized by mean level spacing $\delta_{L}$ and $\delta_{R}$ which are the lowest energy parameters in our problem. These (metallic) dots are interconnected via two tunnel junctions $J_{1}$ and $\mathrm{J}_{2}$ with conductances $G_{t 1}$ and $G_{t 2}$ forming a ringshaped configuration as shown in Fig. 9. The left and

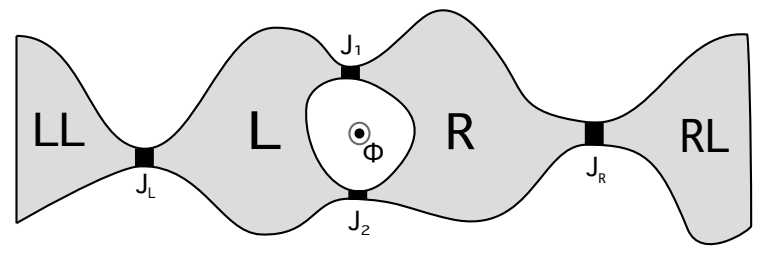

FIG. 9: Two quantum dots with magnetic flux.

right dots are also connected to the leads (LL and RL) respectively via the barriers $\mathrm{J}_{L}$ and $\mathrm{J}_{R}$ with conductances $G_{L}$ and $G_{R}$. We also define the corresponding dimensionless conductances of all four barriers as $g_{t 1,2}=G_{t 1,2} R_{q}$ and $g_{L, R}=G_{t 1,2} R_{q}$, where $R_{q}=2 \pi / e^{2}$ is the quantum resistance unit.

The whole structure is pierced by the magnetic flux $\Phi$ through the hole between two central barriers in such way that electrons passing from left to right through different junctions acquire different geometric phases. Applying a voltage across the system one induces the current which shows $\mathrm{AB}$ oscillations with changing the external flux $\Phi$. Note that in the absence of the magnetic flux the system just reduces to that of two connected in series quantum dots (cf. Fig. 5) which is also subject to weak localization effects. Thus, the model considered here allows to analyze WL and $\mathrm{AB}$ effects within the same formalism to be developed below. The system depicted in Fig. 9 is described by the effective Hamiltonian:

$$
\begin{aligned}
\hat{H}= & \sum_{i, j=L, R} \frac{C_{i j} \hat{\boldsymbol{V}}_{i} \hat{\boldsymbol{V}}_{j}}{2}+\hat{\boldsymbol{H}}_{L L}+\hat{\boldsymbol{H}}_{R L} \\
& +\sum_{j=L, R} \hat{\boldsymbol{H}}_{j}+\hat{\boldsymbol{T}}_{L}+\hat{\boldsymbol{T}}_{R}+\hat{\boldsymbol{T}}
\end{aligned}
$$

where $C_{i j}$ is the capacitance matrix, $\hat{\boldsymbol{V}}_{L(R)}$ is the electric potential operator on the left (right) quantum dot,

$$
\begin{aligned}
& \hat{\boldsymbol{H}}_{L L}=\sum_{\alpha=\uparrow, \downarrow} \int_{L L} d^{3} \boldsymbol{r} \hat{\Psi}_{\alpha, L L}^{\dagger}(\boldsymbol{r})\left(\hat{H}_{L L}-e V_{L L}\right) \hat{\Psi}_{\alpha, L L}(\boldsymbol{r}), \\
& \hat{\boldsymbol{H}}_{R L}=\sum_{\alpha=\uparrow, \downarrow_{R L}} \int^{3} d^{3} \boldsymbol{r} \hat{\Psi}_{\alpha, R L}^{\dagger}(\boldsymbol{r})\left(\hat{H}_{R L}-e V_{R L}\right) \hat{\Psi}_{\alpha, R L}(\boldsymbol{r})
\end{aligned}
$$

are the Hamiltonians of the left and right leads, $V_{L L, R L}$ are the electric potentials of the leads fixed by the external voltage source,

$$
\hat{\boldsymbol{H}}_{j}=\sum_{\alpha=\uparrow, \downarrow} \int_{j} d^{3} \boldsymbol{r} \hat{\Psi}_{\alpha, j}^{\dagger}(\boldsymbol{r})\left(\hat{H}_{j}-e \hat{\boldsymbol{V}}_{j}\right) \hat{\Psi}_{\alpha, j}(\boldsymbol{r})
$$

defines the Hamiltonians of the left $(j=L)$ and right $(j=R)$ quantum dots and

$$
\hat{H}_{j}=\frac{\left(\hat{p}_{\mu}-\frac{e}{c} A_{\mu}(r)\right)^{2}}{2 m}-\mu+U_{j}(r)
$$


is the one-particle Hamiltonian of electron in $j$-th quantum dot with disorder potential $U_{j}(r)$. Electron transfer between the left and the right quantum dots will be described by the Hamiltonian

$$
\hat{\boldsymbol{T}}=\sum_{\alpha=\uparrow, \downarrow} \int_{J_{1}+J_{2}} d^{2} \boldsymbol{r}\left[t(\boldsymbol{r}) \hat{\Psi}_{\alpha, L}^{\dagger}(\boldsymbol{r}) \hat{\Psi}_{\alpha, R}(\boldsymbol{r})+\text { c.c. }\right] .
$$

The Hamiltonian $\hat{\boldsymbol{T}}_{L(R)}$ describing electron transfer between the left dot and the left lead (the right dot and the right lead) is defined analogously.

The real time evolution of the density matrix of our system is described by means of the standard equation

$$
\hat{\rho}(t)=e^{-i \hat{H} t} \hat{\rho}_{0} e^{i \hat{H} t},
$$

where $\hat{H}$ is given by Eq. (63). Let us express the operators $e^{-i \hat{H} t}$ and $e^{i \hat{H} t}$ via path integrals over the fluctuating electric potentials $V_{j}^{F, B}$ defined respectively on the forward and backward parts of the Keldysh contour:

$$
\begin{aligned}
e^{-i \hat{H} t} & =\int D V_{j}^{F} \mathrm{~T} \exp \left\{-i \int_{0}^{t} d t^{\prime} \hat{H}\left[V_{j}^{F}\left(t^{\prime}\right)\right]\right\}, \\
e^{i \hat{H} t} & =\int D V_{j}^{B} \tilde{\mathrm{T}} \exp \left\{i \int_{0}^{t} d t^{\prime} \hat{H}\left[V_{j}^{B}\left(t^{\prime}\right)\right]\right\} .
\end{aligned}
$$

Here $\mathrm{T} \exp (\tilde{\mathrm{T}} \exp )$ stands for the time ordered (antiordered) exponent.

Let us define the effective action of our system

$$
\begin{aligned}
i S\left[V^{F}, V^{B}\right]= & \ln \left(\operatorname { t r } \left[\mathrm{T} \exp \left\{-i \int_{0}^{t} d t^{\prime} \hat{H}\left[V_{j}^{F}\left(t^{\prime}\right)\right]\right\}\right.\right. \\
& \left.\left.\left.\times \hat{\rho}_{0} \tilde{\mathrm{T}} \exp \left\{i \int_{0}^{t} d t^{\prime} \hat{H}\left[V_{j}^{B}\left(t^{\prime}\right)\right]\right\}\right]\right) 66\right)
\end{aligned}
$$

Integrating out the fermionic variables we rewrite the action in the form

$$
i S=i S_{C}+i S_{e x t}+2 \operatorname{Tr} \ln \left[\check{\mathbf{G}}^{-\mathbf{1}}\right] .
$$

Here $S_{C}$ is the standard term describing charging effects, $S_{\text {ext }}$ accounts for an external circuit and

$$
\check{\mathbf{G}}^{-\mathbf{1}}=\left(\begin{array}{cccc}
\hat{G}_{L L}^{-1} & \hat{T}_{L} & 0 & 0 \\
\hat{T}_{L}^{\dagger} & \hat{G}_{L}^{-1} & \hat{T} & 0 \\
0 & \hat{T}^{\dagger} & \hat{G}_{R}^{-1} & \hat{T}_{R} \\
0 & 0 & \hat{T}_{R}^{\dagger} & \hat{G}_{R L}^{-1}
\end{array}\right) .
$$

is the inverse Green-Keldysh function of electrons propagating in the fluctuating fields. Here each quantum dot as well as two leads is represented by the $2 \times 2$ matrix in the Keldysh space:

$$
\hat{G}_{i}^{-1}=\left(\begin{array}{cc}
i \partial_{t}-\hat{H}_{i}+e V_{i}^{F} & 0 \\
0 & -i \partial_{t}+\hat{H}_{i}-e V_{i}^{B}
\end{array}\right)
$$
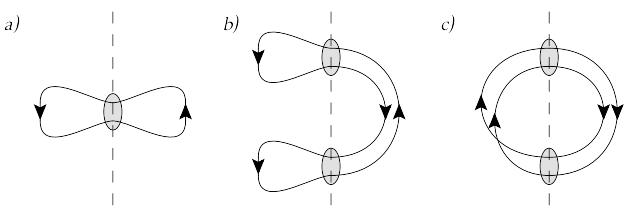

FIG. 10: Diagrammatic representation of different contributions originating from expansion of the effective action in powers of the central barrier transmissions: second order (AES) terms (a) and different fourth order terms (b,c).

\section{Effective action}

Let us expand the exact action $i S$ (67) in powers of $\hat{T}$. Keeping the terms up to the fourth order in the tunneling amplitude, we obtain

$$
\begin{array}{r}
i S \approx i S_{C}+i S_{e x t}+i S_{L}+i S_{R}-2 \operatorname{tr}\left[\hat{G}_{L} \hat{T} \hat{G}_{R} \hat{T}^{\dagger}\right] \\
-\operatorname{tr}\left[\hat{G}_{L} \hat{T} \hat{G}_{R} \hat{T}^{\dagger} \hat{G}_{L} \hat{T} \hat{G}_{R} \hat{T}^{\dagger}\right] .
\end{array}
$$

Here $i S_{L, R}$ are the contributions of isolated dots, the terms $\propto t^{2}$ yield the Ambegaokar-Eckern-Schön (AES) action $10 i S^{A E S}$ described by the diagram in Fig. 10a, and the fourth order terms $\propto t^{4}$ (diagrams in Fig. 10b,c) account for the weak localization correction to the system conductance 24,25 .

It is easy to demonstrate $\underline{\underline{26}}$ that after disorder averaging $i S^{A E S}$ becomes independent of $\Phi$ and, hence, it does not account for the $\mathrm{AB}$ effect investigated here. After averaging the last term in Eq. (70) over realizations of transmission amplitudes and over disorder only the contribution generated by the diagram (c) keeps depending on the magnetic flux and yields 26

$$
\begin{gathered}
i S_{\Phi}^{W L}=-\frac{i g_{t 1} g_{t 2}}{4 \pi^{2} N_{L} N_{R}} \sum_{m, n=1,2} e^{2 i\left(\varphi_{g}^{(n)}-\varphi_{g}^{(m)}\right)} \\
\times \int d \tau_{1} d \tau_{2} \int d t_{1} \ldots d t_{4} C_{L}\left(\tau_{1}\right) C_{R}\left(\tau_{2}\right) \\
\times e^{i\left(\varphi^{+}\left(t_{2}\right)-\varphi^{+}\left(t_{3}\right)+\varphi^{+}\left(t_{4}\right)-\varphi^{+}\left(t_{1}\right)\right)} \sin \frac{\varphi^{-}\left(t_{1}\right)}{2} \\
\times\left[h\left(t_{1}-t_{2}-\tau_{1}\right) e^{i \frac{\varphi^{-}\left(t_{2}\right)}{2}}+\right. \\
\left.\times f\left(t_{1}-t_{2}-\tau_{1}\right) e^{-i \frac{\varphi^{-}\left(t_{2}\right)}{2}}\right] \\
\times\left[h\left(t_{2}-t_{3}-\tau_{2}\right) e^{-i \frac{\varphi^{-}\left(t_{3}\right)}{2}} f\left(t_{3}-t_{4}+\tau_{1}\right)-\right. \\
\left.-f\left(t_{2}-t_{3}-\tau_{2}\right) e^{i \frac{\varphi^{-}\left(t_{3}\right)}{2}} h\left(t_{3}-t_{4}+\tau_{1}\right)\right] \\
\times\left[e^{i \frac{\varphi^{-}\left(t_{4}\right)}{2}} f\left(t_{4}-t_{1}+\tau_{2}\right)+\right. \\
\left.+e^{-i \frac{\varphi^{-}\left(t_{4}\right)}{2}} h\left(t_{4}-t_{1}+\tau_{2}\right)\right] \\
+\left\{L \leftrightarrow R, \varphi^{ \pm} \rightarrow-\varphi^{ \pm}\right\},
\end{gathered}
$$


where $C_{L, R}(t)$ the Cooperons in the left and right dots, $f(t)=\int f_{F}(E) d E / 2 \pi$ is the Fourier transform of the Fermi function $f_{F}(E)$ and $h(t)=\delta(t)-f(t)$. Here we also introduced the geometric phases

$$
\varphi_{g}^{(1,2)}=\frac{e}{c} \int_{L}^{R} d x_{\mu} A_{\mu}(x)
$$

where the integration contour starts in the left dot, crosses the first $\left(\varphi_{g}^{(1)}\right)$ or the second $\left(\varphi_{g}^{(2)}\right)$ junction and ends in the right dot. The difference between these two geometric phases is $\varphi_{g}^{(1)}-\varphi_{g}^{(2)}=2 \pi \Phi / \Phi_{0}$. In addition, we defined the "classical" and the "quantum" components of the fluctuating phase $\varphi^{+}(t)=\left(\varphi_{F}(t)+\varphi_{B}(t)\right) / 2$ and $\varphi^{-}(t)=\varphi_{F}(t)-\varphi_{B}(t)$, where the phases $\varphi_{F, B}(t)=$ $e \int^{t} d \tau\left(V_{R}^{F, B}(\tau)-V_{L}^{F, B}(\tau)\right)$ are defined on the forward and backward parts of the Keldysh contour.

The above expression for the action $S_{\Phi}^{W L}$ (171) fully accounts for coherent oscillations of the system conductance in the lowest non-vanishing order in tunneling. The WL contribution to action of two quantum dots is recovered in exactly the same way ${ }^{24}$. The result is the similar except geometric phases should be omitted and the combination $g_{t 1} g_{t 2}$ should be substituted by $g_{t 1}^{2}$ or $g_{t 2}^{2}$.

\section{Aharonov-Bohm conductance and WL correction}

Let us now evaluate the current $I$ through our system. This current can be split into two parts, $I=I_{0}+\delta I$, where $I_{0}$ is the flux-independent contribution and $\delta I$ is the quantum correction to the current sensitive to the magnetic flux $\Phi$. This correction is determined by the action $i S_{\Phi}^{W L}$, i.e.

$$
\delta I=-e \int \mathcal{D}^{2} \varphi^{ \pm} \frac{\delta S_{\Phi}^{W L}\left[\varphi^{+}, \varphi^{-}\right]}{\delta \varphi^{-}(t)} e^{i S\left[\varphi^{+}, \varphi^{-}\right]} .
$$

In order to evaluate the path integral over the phases $\varphi^{ \pm}$in (73) we restrict our consideration to the most interesting for us metallic limit assuming that dimensionless conductances $g_{L, R}$ are much larger than unity, while the conductances $g_{t 1}$ and $g_{t 2}$ are small as compared to those of the outer barriers, i.e.

$$
g_{L}, g_{R} \gg 1, g_{t 1}, g_{t 2} .
$$

In the limit (74) phase fluctuations can be considered small down to exponentially low energies ${ }^{14,37}$ in which case it suffices to expand both contributions up to the second order $\varphi^{ \pm}$. Moreover, this Gaussian approximation becomes exact $^{15,18,20,21}$ in the limit of fully open left and right barriers with $g_{L, R} \gg 1$. Thus, in the metallic limit (74) the integral (73) remains Gaussian at all relevant energies and can easily be performed.

This task can be accomplished with the aid of the following correlation functions

$$
\left\langle\varphi^{+}(t)\right\rangle=e V t, \quad\left\langle\varphi^{-}(t)\right\rangle=0,
$$

$$
\begin{gathered}
\left\langle\left(\varphi^{+}(t)-\varphi^{+}(0)\right) \varphi^{+}(0)\right\rangle=-F(t), \\
\left\langle\varphi^{+}(t) \varphi^{-}(0)+\varphi^{-}(t) \varphi^{+}(0)\right\rangle=2 i K(|t|), \\
\left\langle\varphi^{+}(t) \varphi^{-}(0)-\varphi^{-}(t) \varphi^{+}(0)\right\rangle=2 i K(t), \\
\left\langle\varphi^{-}(t) \varphi^{-}(0)\right\rangle=0,
\end{gathered}
$$

where the last relation follows directly from the causality principle ${ }^{4}$. Here and below we define $V=V_{R L}-V_{L L}$ to be the transport voltage across our system.

Note that the above correlation functions are well familiar from the so-called $P(E)$-theory $\stackrel{10,38}{ }$ describing electron tunneling in the presence of an external environment which can also mimic electron-electron interactions in metallic conductors. They are expressed in terms of an effective impedance $Z(\omega)$ "seen" by the central barriers $\mathrm{J}_{1}$ and $\mathrm{J}_{2}$

$$
\begin{gathered}
F(t)=e^{2} \int \frac{d \omega}{2 \pi} \operatorname{coth} \frac{\omega}{2 T} \Re[Z(\omega)] \frac{1-\cos (\omega t)}{\omega}, \\
K(t)=e^{2} \int \frac{d \omega}{2 \pi} \Re[Z(\omega)] \frac{\sin (\omega t)}{\omega} .
\end{gathered}
$$

Further evaluation of these correlation functions for our system is straightforward and yields

$$
\begin{gathered}
F(t) \simeq \frac{4}{g}\left(\ln \left|\frac{\sinh (\pi T t)}{\pi T \tau_{R C}}\right|+\gamma\right), \\
K(t) \simeq \frac{2 \pi}{g} \operatorname{sign}(t),
\end{gathered}
$$

where we defined $g=4 \pi / e^{2} Z(0)$ and $\gamma \simeq 0.577$ is the Euler constant. Neglecting the contribution of external leads and making use of the inequality (74) we obtain $g \simeq$ $2 g_{L} g_{R} /\left(g_{L}+g_{R}\right)$. We observe that while $F(t)$ grows with time at any temperature including $T=0$, the function $K(t)$ always remains small and it can be safely ignored in the leading order in $1 / g \ll 1$. After that the Fermi function $f_{F}(E)$ drops out from the final expression for the quantum correction to the current ${ }^{24-26}$. Hence, the amplitude of $\mathrm{AB}$ oscillations is affected by the electronelectron interaction only via the correlation functions for the "classical" component of the Hubbard-Stratonovich phase $\varphi^{+}$.

The expression for the current takes the form

$$
\delta I(\Phi)=-I_{A B} \cos \left(4 \pi \Phi / \Phi_{0}\right)-I_{W L 1}-I_{W L 2},
$$

where the first - flux dependent - term in the right-hand side explicitly accounts for $\mathrm{AB}$ oscillations, while the terms $I_{W L 1,2}$ represent the remaining part of the quantum correction to the current ${ }^{24}$ which does not depend on $\Phi$. 
Let us restrict our attention to the case of two identical quantum dots with volume $\mathcal{V}$, dwell time $\tau_{D}$ and dimensionless conductances $g_{L}=g_{R} \equiv g=4 \pi / \delta \tau_{D}$, where $\delta=1 / \mathcal{V} \nu$ is the dot mean level spacing and $\nu$ is the electron density of states. In this case the Cooperons take the form $C_{L}(t ; \mathbf{x}, \mathbf{y})=C_{R}(t ; \mathbf{x}, \mathbf{y})=(\theta(t) / \mathcal{V}) e^{-t / \tau_{D}}$. We obtain 26

$$
\begin{gathered}
I_{A B}=\frac{e^{2} g_{t 1} g_{t 2} \delta^{2} V}{4 \pi^{3}} \int_{0}^{\infty} d \tau_{1} d \tau_{2} e^{-\frac{\tau_{1}+\tau_{2}}{\tau_{D}}-\mathcal{F}\left(\tau_{1}, \tau_{2}\right)} . \\
I_{W L 1,2}=\frac{e^{2} g_{t 1,2}^{2} \delta^{2} V}{8 \pi^{3}} \int_{0}^{\infty} d \tau_{1} d \tau_{2} e^{-\frac{\tau_{1}+\tau_{2}}{\tau_{D}}-\mathcal{F}\left(\tau_{1}, \tau_{2}\right)} .
\end{gathered}
$$

where $\mathcal{F}=2 F\left(\tau_{1}\right)+2 F\left(\tau_{2}\right)-F\left(\tau_{1}-\tau_{2}\right)-F\left(\tau_{1}+\tau_{2}\right)$.

In the absence of electron-electron interactions this formula yields $I_{A B}^{(0)}=4 e^{2} g_{t 1} g_{t 2} V /\left(\pi g^{2}\right)$. In order to account for the effect of interactions we substitute Eq. (82) into Eq. (85). Performing time integrations at high enough temperatures we obtain

$$
\frac{I_{A B}}{I_{A B}^{(0)}}=\left\{\begin{array}{lc}
e^{-\frac{8 \gamma}{g} \frac{\left(2 \pi T \tau_{R C}\right)^{8 / g}}{1+4 \pi T \tau_{D} / g},}, & \tau_{D}^{-1} \lesssim T \lesssim \tau_{R C}^{-1}, \\
\frac{1}{2 \tau_{D}}\left(\frac{g \tau_{R C}}{T}\right)^{1 / 2}, & \tau_{R C}^{-1} \lesssim T,
\end{array}\right.
$$

while in the low temperature limit we find

$$
\frac{I_{A B}}{I_{A B}^{(0)}}=e^{-\frac{8 \gamma}{g}}\left(\frac{2 \tau_{R C}}{\tau_{D}}\right)^{8 / g}, \quad T \lesssim \tau_{D}^{-1} .
$$

Essentially the same results follow for $I_{W L 1,2}$. These results demonstrate that interaction-induced suppression of both $\mathrm{AB}$ oscillations and WL corrections in metallic dots with $\tau_{R C} \ll \tau_{D}$ persists down to $T=0$. The fundamental reason behind this suppression is that the interaction of an electron with an effective environment (produced by other electrons) effectively breaks down the time-reversal symmetry and, hence, causes both dissipation and dephasing for interacting electrons down to $T=0$. . In this respect it is also important to point out a deep relation between interaction-induced electron decoherence and the $P(E)$-theory 10,38 which was already emphasized elsewhere $24-26$.

\section{Arrays of quantum dots and diffusive conductors}

One of the main conclusions reached above is that the electron decoherence time is fully determined by fluctuations of the phase fields $\varphi^{+}$(and the correlation function $F(t)$ ), whereas the phases $\varphi^{-}$(and the response function $K(t))$ are irrelevant for $\tau_{\varphi}$ causing only a weak Coulomb correction to $G_{W L}$. This conclusion is general being independent of a number of scatterers in our system. Note that exactly the same conclusion was already reached in the case of diffusive metals by means of a different approach $\stackrel{4}{*}$. Thus, in order to evaluate the decoherence time for interacting electrons in arrays of quantum dots it is sufficient to account for the fluctuating fields $V^{+}$ totally ignoring the fields $V^{-}$. The corresponding calculation is presented below.

\section{1. $1 d$ structures}

Let us consider a 1D array of $N-1$ quantum dots by $N$ identical barriers as shown in Fig. 1. For simplicity, we will stick to the case of identical barriers (with dimensionless conductance $g \gg 1$ and Fano factor $\beta$ ) and identical quantum dots (with mean level spacing $\delta$ and dwell time $\tau_{D}=2 \pi / \delta g$ ). The WL correction to the system conductance has the form (see Eq. (36)):

$$
\begin{aligned}
G_{W L}= & -\frac{e^{2} g \delta}{4 \pi^{2} N^{2}} \sum_{n=1}^{N} \int_{0}^{\infty} d t \\
& \times\left\{\beta\left[C_{n-1, n}(t)+C_{n, n-1}(t)\right]\right. \\
& \left.+(1-\beta)\left[C_{n n}(t)+C_{n-1, n-1}(t)\right]\right\} .
\end{aligned}
$$

The Cooperon $C_{n m}(t)$ is determined from a discrete version of the diffusion equation. For non-interacting electrons and in the absence of the magnetic field this equation reads

$$
\frac{\partial C_{n m}}{\partial t}+\frac{2 C_{n m}-C_{n-1, m}-C_{n+1, m}}{2 \tau_{D}}=\delta_{n m} \delta(t) .
$$

The boundary conditions for this equation are $C_{n m}=0$ as long as the index $n$ or $m$ belongs to one of the bulk electrode. The solution of Eq. (90) with these boundary conditions can easily be obtained. We have

$$
C_{n m}^{(0)}(t)=\frac{2}{N} \sum_{q=1}^{N-1} \int \frac{d \omega}{2 \pi} e^{-i \omega t} \frac{\sin \frac{\pi q n}{N} \sin \frac{\pi q m}{N}}{-i \omega+\frac{1-\cos \frac{\pi q}{N}}{\tau_{D}}} .
$$

This solution can be represented in the form $C_{n m}^{(0)}(t)=$ $C_{n-m}^{\text {bulk }}(t)-C_{n+m}^{\text {bulk }}(t)$, where

$$
C_{n-m}^{\mathrm{bulk}}(t)=\frac{1}{N} \sum_{q=1}^{N-1} \int \frac{d \omega}{2 \pi} e^{-i \omega t} \frac{\cos \frac{\pi q(n-m)}{N}}{-i \omega+\frac{1-\cos \frac{\pi q}{N}}{\tau_{D}}} .
$$

In the limit of large $N$ the term $C_{n+m}^{\text {bulk }}(t)$ can be safely ignored and we obtain $C_{n m}(t) \approx C_{n-m}^{\text {bulk }}(t)$. Let us express the contribution $C_{n-m}^{\text {bulk }}(t)$ as a sum over the integer valued paths $\nu(\tau)$, which start in the $m$-th dot and end in the $n$-th one (i.e. $\nu(0)=m, \nu(t)=n$ ) jumping from one dot to another at times $t_{j}$. This expression can be recovered if one expands Eq. (92) in powers of $\tau_{D}^{-1} \cos [\pi q / N]$ with subsequent summation over $q$ in every order of this expansion. Including additional phase factors acquired by electrons in the presence of the fluctuating fields $V_{\nu}^{+}$, 
we obtain

$$
\begin{aligned}
& C_{n m}(t)=\left.\sum_{k=|n-m|}^{\infty} \sum_{\nu(\tau)}\right|_{\nu(0)=m} ^{\nu(t)=n} \\
& \times \frac{1}{\left(2 \tau_{D}\right)^{k}} \int_{0}^{t} d t_{k} \int_{0}^{t_{k}} d t_{k-1} \ldots \int_{0}^{t_{3}} d t_{2} \int_{0}^{t_{2}} d t_{1} \\
& \times e^{-\frac{t-t_{k}}{\tau_{D}}} e^{-\frac{t_{k}-t_{k-1}}{\tau_{D}}} \ldots e^{-\frac{t_{2}-t_{2}}{\tau_{D}}} e^{-\frac{t_{2}-t_{1}}{\tau_{D}}} e^{-\frac{t_{1}}{\tau_{D}}} \\
& \times \exp \left\{i \int_{0}^{t} d \tau\left[e V_{\nu(\tau)}^{+}(\tau)-e V_{\nu(t-\tau)}^{+}(\tau)\right]\right\} .
\end{aligned}
$$

Averaging over Gaussian fluctuations of voltages $V^{+}$ and utilizing the symmetry of the voltage correlator $\left\langle V_{\nu_{1}}^{+}\left(\tau_{1}\right) V_{\nu_{2}}^{+}\left(\tau_{2}\right)\right\rangle=\left\langle V_{\nu_{2}}^{+}\left(\tau_{1}\right) V_{\nu_{1}}^{+}\left(\tau_{2}\right)\right\rangle$, we get

$$
\begin{aligned}
& C_{n m}(t)=\left.\sum_{k=|n-m|}^{\infty} \sum_{\nu(\tau)}\right|_{\nu(0)=m} ^{\nu(t)=n} \\
& \times \frac{e^{-t / \tau_{D}}}{\left(2 \tau_{D}\right)^{k}} \int_{0}^{t} d t_{k} \int_{0}^{t_{k}} d t_{k-1} \ldots \int_{0}^{t_{3}} d t_{2} \int_{0}^{t_{2}} d t_{1} \\
& \times \exp \left\{-e^{2} \int_{0}^{t} d \tau_{1} \int_{0}^{t} d \tau_{2}\left[\left\langle V_{\nu\left(\tau_{1}\right)}^{+}\left(\tau_{1}\right) V_{\nu\left(\tau_{2}\right)}^{+}\left(\tau_{2}\right)\right\rangle\right.\right. \\
& \left.\left.-\left\langle V_{\nu\left(\tau_{1}\right)}^{+}\left(\tau_{1}\right) V_{\nu\left(t-\tau_{2}\right)}^{+}\left(\tau_{2}\right)\right\rangle\right]\right\} .
\end{aligned}
$$

The correlator of voltages can be derived with the aid of the $\sigma$-model approach developed in Sec. 2 of this paper. Integrating over Gaussian fluctuations of the $Q$ fields one arrives at the quadratic action for the fluctuating fields $V^{+}$which has the form

$$
\begin{aligned}
& i S=\frac{2 i}{N} \sum_{q=1}^{N} \int \frac{d \omega}{2 \pi}\left[4 C\left(1-\cos \frac{\pi q}{N}\right)+C_{g}\right. \\
& \left.+\frac{g \tau_{D} e^{2}}{\pi} \frac{1-\cos \frac{\pi q}{N}}{-i \omega \tau_{D}+1-\cos \frac{\pi q}{N}}\right] V_{q}^{+}(\omega) V_{q}^{-}(-\omega) \\
& -\frac{2}{N} \sum_{q=1}^{N} \int \frac{d \omega}{2 \pi} \frac{g \tau_{D}^{2} e^{2}}{\pi} \frac{\left(1-\cos \frac{\pi q}{N}\right) \omega \operatorname{coth} \frac{\omega}{2 T}}{\omega^{2} \tau_{D}^{2}+\left(1-\cos \frac{\pi q}{N}\right)^{2}} \\
& \times V_{q}^{-}(\omega) V_{q}^{-}(-\omega) .
\end{aligned}
$$

Here we defined

$$
V_{q}^{ \pm}(\omega)=\sum_{n=1}^{N-1} \int d t \sin \frac{\pi q}{N} e^{i \omega t} V_{n}^{ \pm}(t)
$$

The action (95) determines the expressions for both correlators $\left\langle V^{+} V^{+}\right\rangle$( $F$-function) and $\left\langle V^{+} V^{-}\right\rangle$( $K$-function) responsible respectively for decoherence and Coulomb blockade correction to WL. Since our aim is to describe electron decoherence, only the first out of these two cor- relation functions is of importance for us here. It reads

$$
\begin{aligned}
& \left\langle V_{n}^{+}\left(t_{1}\right) V_{m}^{+}\left(t_{2}\right)\right\rangle=\frac{2}{N} \sum_{q=1}^{N-1} \int \frac{d \omega}{2 \pi} e^{-i \omega\left(t_{1}-t_{2}\right)} \\
& \times \frac{\frac{g e^{2}}{\pi}\left(1-\cos \frac{\pi q}{N}\right) \sin \frac{\pi q n}{N} \sin \frac{\pi q m}{N}}{\left|4 C\left(1-\cos \frac{\pi q}{N}\right)+C_{g}+\frac{g \tau_{D} e^{2}}{\pi} \frac{1-\cos \frac{\pi q}{N}}{-i \omega \tau_{D}+1-\cos \frac{\pi q}{N}}\right|^{2}} \\
& \times \frac{\tau_{D}^{2} \omega \operatorname{coth} \frac{\omega}{2 T}}{\omega^{2} \tau_{D}^{2}+\left(1-\cos \frac{\pi q}{N}\right)^{2}} .
\end{aligned}
$$

In the continuous limit $N \gg 1$ and for sufficiently low frequencies $\omega \ll 1 / \tau_{D}$ both correlators $\left\langle V^{+} V^{+}\right\rangle$and $\left\langle V^{+} V^{-}\right\rangle$defined by Eq. (95) reduce to those of a diffusive metal $\stackrel{4}{ }$.

To proceed let us consider diffusive paths $\nu(\tau)$, in which case one has

$$
\begin{aligned}
\left\langle V_{\nu\left(\tau_{1}\right)}^{+}\left(\tau_{1}\right) V_{\nu\left(\tau_{2}\right)}^{+}\left(\tau_{2}\right)\right\rangle \approx & \frac{1}{N-1} \sum_{n, m=1}^{N-1}\left\langle V_{n}^{+}\left(\tau_{1}\right) V_{m}^{+}\left(\tau_{2}\right)\right\rangle \\
& \times D_{n m}\left(\left|\tau_{1}-\tau_{2}\right|\right)
\end{aligned}
$$

where $D_{n m}(\tau)$ is the diffuson. For $H \rightarrow 0$ it exactly coincides with the Cooperon for non-interacting electrons (91), $D_{n m}(t)=C_{n, m}^{(0)}(t)$, i.e.

$$
D_{n m}(t)=\frac{2}{N} \sum_{q=1}^{N-1} \int \frac{d \omega}{2 \pi} e^{-i \omega t} \frac{\sin \frac{\pi q n}{N} \sin \frac{\pi q m}{N}}{-i \omega+\frac{1-\cos \frac{\pi q}{N}}{\tau_{D}}} .
$$

Substituting Eq. (98) into (94), we obtain

$$
C_{n m}(t) \approx C_{n m}^{(0)}(t) e^{-\mathcal{F}(t)},
$$

where

$$
\begin{aligned}
\mathcal{F}(t)= & \frac{e^{2}}{N-1} \sum_{n, m=1}^{N-1} \int_{0}^{t} d t_{1} d t_{2}\left\langle V_{n}^{+}\left(t_{1}\right) V_{m}^{+}\left(t_{2}\right)\right\rangle \\
& \left.\times\left[D_{n m}\left(\left|t_{1}-t_{2}\right|\right)-D_{n m}\left(\left|t-t_{1}-t_{2}\right|\right)\right] 101\right)
\end{aligned}
$$

is the function which controls the Cooperon decay in time, i.e. describes electron decoherence for our $1 \mathrm{~d}$ array of quantum dots. The WL correction $G_{W L}$ in the presence of electron-electron interactions is recovered by substituting the result (100) into Eq. (89).

Since the behavior of the latter formula was already analyzed in details earlier there is no need to repeat this analysis here. The dephasing time $\tau_{\varphi}$ can be extracted from the equation $\mathcal{F}\left(\tau_{\varphi}\right)=1$. From Eq. (101) with a good accuracy we obtain

$$
\frac{1}{\tau_{\varphi}}=\frac{e^{2}}{N-1} \sum_{n, m=1}^{N-1} \int d \tau\left\langle V_{n}^{+}(\tau) V_{m}^{+}(0)\right\rangle D_{n m}(\tau) .
$$

Combining this formula with Eqs. (97) and (99), in the most interesting limit $T \rightarrow 0$ and for $\tau_{D} \gg R\left(4 C+C_{g}\right)$ we find

$$
\frac{1}{\tau_{\varphi 0}}=\frac{1}{2 g \tau_{D}(N-1)} \sum_{q=1}^{N-1} \ln \frac{2 e^{2}}{\delta\left(4 C\left(1-\cos \frac{\pi q}{N}\right)+C_{g}\right)},
$$


which yields

$$
\tau_{\varphi 0}=\frac{2 g \tau_{D}}{\ln \left(4 \tilde{E}_{C} / \delta\right)}=\frac{4 \pi}{\delta \ln \left(4 \tilde{E}_{C} / \delta\right)},
$$

where $\tilde{E}_{C}=e^{2} / 2 C_{g}$ for $C_{g} \gg C$ and $\tilde{E}_{C}=e^{2} / 4 C$ in the opposite case $C_{g} \ll C$.

In order to determine the dephasing length $L_{\varphi}=$ $\sqrt{D \tau_{\varphi}}$ let us define the diffusion coefficient

$$
D=\frac{d^{2}}{2 \tau_{D}}=\frac{d^{2} g \delta}{4 \pi}
$$

where $d \equiv \mathcal{V}^{1 / 3}$ is the average dot size. Combining Eqs. (103) and (104), at $T=0$ we obtain

$$
L_{\varphi 0}=\sqrt{D \tau_{\varphi 0}}=d \sqrt{g / \ln \left(4 \tilde{E}_{C} / \delta\right)} .
$$

At non-zero $T$ thermal fluctuations provide an additional contribution to the dephasing rate $1 / \tau_{\varphi}$. Again substituting Eqs. (97) and (99) into (102), we get

$$
\frac{1}{\tau_{\varphi}(T)} \simeq \frac{1}{\tau_{\varphi 0}}+\frac{\pi T}{3 g} \min \left\{N, N_{\varphi}\right\}
$$

where $N_{\varphi}=L_{\varphi} / d \sim \sqrt{\tau_{\varphi} / \tau_{D}}$ is the number of quantum dots within the length $L_{\varphi}$. We observe that for sufficiently small $N<N_{\varphi}$ (but still $N \gg 1$ ) the dephasing rate increases linearly both with temperature and with the number $N$. At larger $N>\sqrt{g / \ln \left[4 \tilde{E}_{C} / \delta\right]}$ and/or at high enough temperatures $N_{\varphi}$ becomes smaller than $N$ and Eq. (106) for $\tau_{\varphi}$ should be resolved self-consistently. In this case we obtain

$$
\tau_{\varphi} \simeq\left(3 g \sqrt{\tau_{D}} / \pi T\right)^{2 / 3}
$$

thus reproducing the well known result ${ }^{29}$. Eq. (106) also allows to estimate the temperature $T^{*} \simeq$ $2 \pi g /\left[\tau_{\varphi 0} \min \left\{N, N_{\varphi}\right\}\right]$ at which the crossover to the temperature-independent regime (103) occurs. We find

$$
\begin{aligned}
& T^{*} \simeq \frac{3 \ln \left[4 \tilde{E}_{C} / \delta\right]}{2 \pi N \tau_{D}}, \quad N \lesssim \sqrt{\frac{g}{\ln \left[4 \tilde{E}_{C} / \delta\right]}}, \\
& T^{*} \simeq \frac{3 \ln ^{3 / 2}\left[4 \tilde{E}_{C} / \delta\right]}{2 \pi \tau_{D} \sqrt{g}}, \quad N \gtrsim \sqrt{\frac{g}{\ln \left[4 \tilde{E}_{C} / \delta\right]}} .
\end{aligned}
$$

\section{Good metals and granular conductors}

The above analysis and conclusions can be generalized further to the case $2 \mathrm{~d}$ and $3 \mathrm{~d}$ structures. This generalization is absolutely straightforward (see, e.g. $\stackrel{23}{=}$ ) and therefore is not elaborated here. At $T \rightarrow 0$ one again arrives at the same result for $\tau_{\varphi 0}$ (103).

Now we discuss the relation between our present results and those derived earlier for weakly disordered metals by means of a different approach $\stackrel{4}{*}$. Let us express the dot mean level spacing via the average dot size $d$ as $\delta=$ $1 / N_{0} d^{3}$ (where $N_{0}=m p_{F} / 2 \pi^{2}$ is the electron density of states at the Fermi level). Then we obtain

$$
D=\frac{g}{4 \pi N_{0} d} .
$$

Below we consider two different physical limits of $(a)$ good metals and $(b)$ strongly disordered (granular) conductors. For the model $(a)$ we assume that quantum dots are in a good contact with each other. In this case $g$ scales linearly with the contact area $\mathcal{A}=\gamma d^{2}$, where $\gamma$ is a numerical factor of order (typically smaller than) one which particular value depends on geometry. For weakly disordered metals most conducting channels in such contacts can be considered open. Hence, $g=p_{F}^{2} \mathcal{A} / 2 \pi$ and

$$
D=\gamma v_{F} d / 4,
$$

i.e. $D \propto d$. If most channels are not fully transparent, then the factor $\gamma$ in (110) also accounts for their transmissions. Comparing Eq. (110) with the standard definition of $D$ for a bulk diffusive conductor, $D=v_{F} l / 3$, we immediately observe that within our model the average dot size is comparable to the elastic mean free path, $l \sim \gamma d$, as it should be for weakly disordered metals.

Expressing $\tau_{\varphi 0}$ (103) via $D$, in this limit we get

$$
\tau_{\varphi 0}=\frac{64}{\pi \gamma^{3}} \frac{m^{2}}{v_{F}^{2}} \frac{D^{3}}{\ln \left(D / D_{c 1}\right)},
$$

where $m$ is the electron mass and $D_{c 1}$ is constant which depends on $\tilde{E}_{C}$. Estimating, e.g., $\tilde{E}_{C} \approx e^{2} / 2 d$, one obtains $D_{c 1}^{-1}=4 e \sqrt{2 N_{0}} / \gamma v_{F}$.

Note that apart from an unimportant numerical prefactor and the logarithm in the denominator of Eq. (111) the latter result for $\tau_{\varphi 0}$ coincides with that derived for a bulk diffusive metal within the framework of a completely different approach ${ }^{4}$. Within that approach local properties of the model remain somewhat ambiguous and, hence, in the corresponding integrals in $\underline{\underline{4}}$ we could not avoid using an effective high frequency cutoff procedure. This cutoff yields the correct leading dependence $\tau_{\varphi 0} \propto D^{3}$ and it only does not allow to recover an additional logarithmic dependence on $D$ in (111). Our present approach is divergence-free and, hence, it does not require any cutoffs.

We can also add that Eq. (103) also agrees with our earlier results $\underline{4}$ derived for quasi- $1 \mathrm{~d}$ and quasi- $2 \mathrm{~d}$ metallic conductors. Provided the transversal size $a$ of our array is smaller than $d$ one should set $\mathcal{A} \sim d a$ for $2 \mathrm{~d}$ and $\mathcal{A} \sim a^{2}$ for $1 \mathrm{~d}$ conductors. Then Eq. (103) yields $\tau_{\varphi 0} \propto D^{2} / \ln D$ and $\tau_{\varphi 0} \propto D / \ln D$ respectively in $2 \mathrm{~d}$ and $1 \mathrm{~d}$ cases. Up to the factor $\ln D$ these dependencies coincide with ones derived previously $\underline{\underline{4}}$.

Now let us turn to the model (b) of strongly disordered and/or granular conductors. In contrast to the situation $(a)$, we will assume that the contact between dots (grains) is rather poor, and inter-grain electron transport 


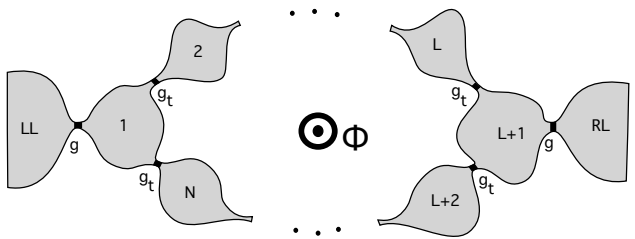

FIG. 11: Ring composed of $N$ quantum dots

may occur only via limited number of conducting channels. In this case the average dimensionless conductance $g$ can be approximated by some $\mathcal{A}$-independent constant $g=g_{c}$. Substituting $g_{c}$ instead of $g$ into Eq. (109) we observe that in the case of strongly disordered structures one can expect $D \propto 1 / d$. Accordingly, for $\tau_{\varphi 0}$ (103) one finds

$$
\tau_{\varphi 0}=\frac{g_{c}^{3}}{32 \pi^{2} N_{0}^{2} D^{3} \ln \left(D_{c 2} / D\right)},
$$

where $D_{c 2}$ again depends on $\tilde{E}_{C}$. For $\tilde{E}_{C} \approx e^{2} / 2 d$ we have $D_{c 2}^{-1}=2 \pi \sqrt{2 N_{0}} / e g_{c}$. Hence, the dependence of $\tau_{\varphi 0}$ on $D$ for strongly disordered or granular conductors (112) is it qualitatively different from that for sufficiently clean metals (111).

One can also roughly estimate the crossover between the regimes $(a)$ and $(b)$ by requiring the values of $D=$ $\gamma v_{F} d / 4$ (110) and $D=g_{c} / 4 \pi N_{0} d$ to be of the same order. This condition yields $\left(p_{F} d\right)^{2} \sim 2 \pi g_{c} / \gamma$, and we arrive at the estimate for $D$ at the crossover

$$
D \approx \frac{0.6 \hbar}{m} \sqrt{\frac{g_{c}}{\gamma}} .
$$

Here we restored the Planck constant $\hbar$ set equal to unity elsewhere in our paper.

\section{Ring composed of quantum dots}

Now let us turn to a ring-shaped nanostructure as shown in Fig. 11. For simplicity we will consider the case of identical quantum dots (with mean level spacing $\delta$ and dwell time $\tau_{D}=2 \pi /(g \delta)$ ) coupled by junctions with conductances $g_{t}$ and the Fano-factor $\beta_{t}$. Leads are coupled to the ring at the dots with numbers 1 and $L+1$ by junctions with conductance $g$. The interference correction to the conductance of n-th junction $\delta G_{n}$ was already derived in Sec. 2 by means of the non-linear sigma-model approach. We obtain

$$
\begin{array}{r}
\delta G_{n}=-\frac{e^{2} g_{t} \delta}{4 \pi^{2}} \int_{0}^{\infty} d t\left[\beta_{t} C_{n, n+1}(t) e^{\frac{4 \pi i \Phi}{N \Phi_{0}}}+\right. \\
+\left(1-\beta_{t}\right)\left(C_{n, n}(t)+C_{n+1, n+1}(t)\right)+ \\
\left.+\beta_{t} C_{n+1, n}(t) e^{-\frac{4 \pi i \Phi}{N \Phi_{0}}}\right],
\end{array}
$$

where $C_{m, n}(t)$ is the Cooperon. The quantum correction to conductance of the whole system can be obtained with the aid of the Kirchhoff's law. For the case $N g \ll g_{t}$ considered here one finds

$$
\delta G=\frac{N L(N-L) g^{2}}{\left(2 N g_{t}+L(N-L) g\right)^{2}} \delta g \approx \frac{L(N-L) g^{2}}{4 N g_{t}^{2}} \delta G_{n} .
$$

Further procedure is analogous to that implemented above for $1 \mathrm{~d}$ arrays. The main difference of the present ring-shaped geometry just concerns the form of diffusons $D_{m n}(t)$, cooperons $C_{m n}^{(0)}(t)$ and the fluctuating voltage correlators $F_{m n}(t)=\left\langle V_{m}^{+}(t) V_{n}^{+}(0)\right\rangle_{V^{+}}$. We obtain

$$
\begin{gathered}
D_{m n}(t)=\frac{\tau_{D}}{N} \sum_{q=1}^{N} \int \frac{d \omega}{2 \pi} \frac{e^{-i \omega t+\frac{2 \pi i q}{N}(m-n)}}{-i \omega \tau_{D}+\epsilon(q)}, \\
C_{m n}^{(0)}(t)=\frac{\tau_{D}}{N} \sum_{q=1}^{N} \int \frac{d \omega}{2 \pi} \frac{e^{-i \omega t+\frac{2 \pi i q}{N}(m-n)}}{-i \omega \tau_{D}+\epsilon\left(q-2 \Phi / \Phi_{0}\right)},
\end{gathered}
$$

and

$$
F_{m n}(t)=\frac{\tau_{D}}{N} \sum_{q=1}^{N} \int \frac{d \omega}{2 \pi} e^{-i \omega t} \omega \operatorname{coth} \frac{\omega}{2 T} \frac{f(q) e^{\frac{2 \pi i q}{N}(m-n)}}{\omega^{2} \tau_{D}^{2}+\varepsilon^{2}(q)},
$$

where

$$
\begin{gathered}
f(q)=\frac{g_{t} \tau_{D} e^{2}}{\pi} \frac{\epsilon(q)}{\left(4 C \epsilon(q)+C_{g}\right)^{2}}, \\
\varepsilon(q)=\epsilon(q)+\frac{g_{t} \tau_{D} e^{2}}{\pi} \frac{\epsilon(q)}{4 C \epsilon(q)+C_{g}}
\end{gathered}
$$

and $\epsilon(q)=1-\cos \frac{2 \pi q}{N}$. As above, here $C$ and $C_{g}$ denote respectively the junction and the dot capacitances.

The above equations are sufficient to evaluate the function $\mathcal{F}(t)$ in a general form. Here we are primarily interested in $\mathrm{AB}$ oscillations and, hence, we only need to account for the flux-dependent contributions determined by the electron trajectories which fully encircle the ring at least once. Obviously, one such traverse around the ring takes time $t \geq N^{2} \tau_{D}$. Hence, the behavior of the function $\mathcal{F}(t)$ only at such time scales needs to be studied for our present purposes. In this long time limit $\mathcal{F}(t)$ is a linear function of time with the corresponding slope

$$
\begin{aligned}
& \mathcal{F}^{\prime}\left(t \geq N^{2} \tau_{D}\right) \approx \\
& \approx \frac{2 e^{2} \tau_{D}^{2}}{N} \sum_{q=1}^{N-1} \int \frac{d \omega}{2 \pi} \frac{f(q) \epsilon(q) \omega \operatorname{coth} \frac{\omega}{2 T}}{\left(\omega^{2} \tau_{D}^{2}+\epsilon^{2}(q)\right)\left(\omega^{2} \tau_{D}^{2}+\varepsilon^{2}(q)\right)}(1
\end{aligned}
$$

This observation implies that at such time scales electronelectron interactions yield exponential decay of the Cooperon in time

$$
C_{m n}(t) \approx C_{m n}^{(0)}(t) e^{-\frac{t}{\tau_{\phi}}}
$$


where

$$
\frac{1}{\tau_{\phi}}=\mathcal{F}^{\prime}\left(t \geq N^{2} \tau_{D}\right)
$$

is the effective dephasing time for our problem. In the case $C_{g} \gg C$ and $\tau_{D} \gg \tau_{R C} \equiv 2 \pi C_{g} /\left(e^{2} g_{t}\right)$ from Eq. (124) we obtain

$$
\frac{1}{\tau_{\phi}}= \begin{cases}\frac{\delta}{\pi} \ln _{\frac{4 E_{C}}{\delta}} & T \ll 1 / N \tau_{D}, \\ \frac{\pi N T}{3 g_{t}} & T \gg 1 / N \tau_{D},\end{cases}
$$

where $E_{C}=e^{2} /\left(2 C_{g}\right)$. These expressions are, of course, fully consistent with the results derived above in the case of $1 \mathrm{~d}$ chains of quantum dots and weakly disordered diffusive conductors, cf. also $\underline{\underline{4}}$.

Let us emphasize again that the above results for $\mathcal{F}(t)$ apply at sufficiently long times which is appropriate in the case of $\mathrm{AB}$ conductance oscillations. At the same time, other physical quantities, such as, e.g., weak localization correction to conductance can be determined by the function $\mathcal{F}(t)$ at shorter time scales. Our general results allow to easily recover the corresponding behavior as well. For instance, at $T \gg \tau_{D}$ and $t \ll N^{2} \tau_{D}$ we get

$$
\mathcal{F}(t) \approx \frac{4 T}{3 g_{t}}\left(\frac{2 \pi}{\tau_{D}}\right)^{1 / 2} t^{3 / 2}+\ldots
$$

in agreement with the results 25 . This expression yields the well known dependence $\tau_{\phi} \propto T^{-2 / 3}$ which - in contrast to Eq. (124) - does not depend on $N$ and remains applicable in the high temperature limit.

To proceed further let us integrate the expression for the Cooperon over time. We obtain

$$
\begin{aligned}
& \int_{0}^{\infty} C_{m n}(t) d t= \\
= & \frac{\tau_{D}}{N} \sum_{q=1}^{N} \frac{e^{\frac{2 \pi i q}{N}(m-n)}}{\epsilon\left(q-2 \Phi / \Phi_{0}\right)+\tau_{D} / \tau_{\phi}+g /\left(g_{t} N\right)},
\end{aligned}
$$

where the term $g /\left(g_{t} N\right)$ in the denominator accounts for the effect of external leads and remains applicable as long as $N g \ll g_{t}$. Combining Eqs. (114), (115) and (126) after summation over $q$ we arrive at the final result

$$
\begin{aligned}
& \delta G^{A B}=\frac{e^{2} L(N-L) g^{2}}{2 \pi N g_{t}^{2}} \\
& \quad \times \frac{\left(\beta_{t} \alpha+1-\beta_{t}\right)\left(z^{-N}-\cos \left(4 \pi \Phi / \Phi_{0}\right)\right)}{\sqrt{\alpha^{2}-1}\left(z^{N}+z^{-N}-2 \cos \left(4 \pi \Phi / \Phi_{0}\right)\right)},
\end{aligned}
$$

where $\alpha=1+\frac{\tau_{D}}{\tau_{\phi}}+\frac{g}{g_{t} N}$ and $z=\alpha+\sqrt{\alpha^{2}-1}$. This equation with Eq. (124) fully determines $\mathrm{AB}$ oscillations of conductance in nanorings composed of metallic quantum dots in the presence of electron-electron interactions.

Expanding Eq. (127) in Fourier series we obtain

$$
\delta G^{A B}=\sum_{k=1}^{\infty} \delta G^{(k)} \cos \left(4 \pi k \Phi / \Phi_{0}\right)
$$

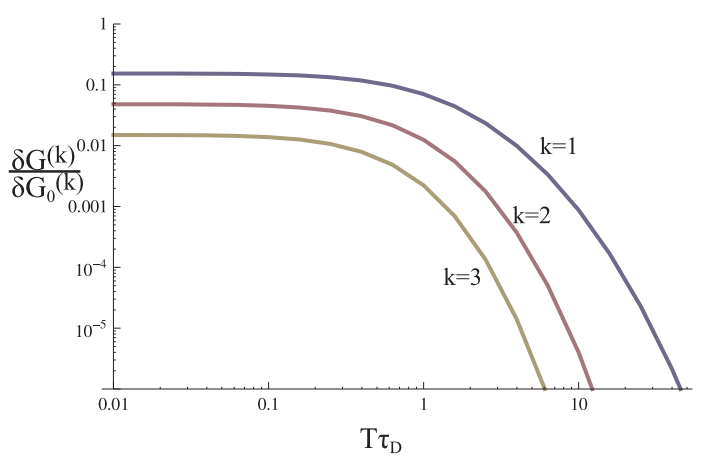

FIG. 12: Temperature dependence of the first three harmonics of AB conductance for $g_{t}=500, g=30, N=10, \beta_{t}=1$ and $\tau_{D} / \tau_{R C}=120$.

where

$$
\delta G^{(k)}=-\frac{e^{2} L(N-L) g^{2}\left(\beta_{t} \alpha+1-\beta_{t}\right)}{2 \pi N g_{t}^{2} \sqrt{\alpha^{2}-1}} z^{-N|k|}
$$

In the limit $\tau_{\phi} \gg \tau_{D}$ we have $z \approx 1+\sqrt{2 \tau_{D} / \tau_{\phi}}+\ldots$, hence $\delta G^{(k)}$ behaves as

$$
\delta G^{(k)} \propto e^{-N|k| \sqrt{\frac{2 \tau_{D}}{\tau_{\phi}}}},
$$

i.e. at hight temperatures $\log |\delta G|$ scales with $N$ as $N^{3 / 2}$ while at low temperatures it scales as $N$. The temperature dependence of the first three harmonics of $\mathrm{AB}$ conductance in the presence of electron-electron interactions is depicted in Fig. 12.

The results obtained here allow to formulate quantitative predictions regading the effect of electron-electron interactions on Aharonov-Bohm oscillations of conductance for a wide class of disordered nanorings embraced by our model. Of particular interest is the situation of large number of dots $N \gg 1$ which essentially mimics the behavior of diffusive nanostructures. In order to establish a direct relation to this important case it is instructive to introduce the diffusion coefficient $D=d^{2} /\left(2 \tau_{D}\right)$ and define the electron density of states $\nu=1 /\left(d^{3} \delta\right)$, where $d$ is a linear dot size. Then we obtain with exponential accuracy:

$$
\delta G^{(k)} \sim \begin{cases}e^{-|k|\left(\mathcal{L} / \mathcal{L}_{\phi}\right)} & T \ll D /(\mathcal{L} d), \\ e^{-|k|\left(\mathcal{L} / \mathcal{L}_{\phi}\right)^{3 / 2}} & T \gg D /(\mathcal{L} d) .\end{cases}
$$

Here we introduced the ring perimeter $\mathcal{L}=N d$ and the effective decoherence length

$$
\mathcal{L}_{\phi}= \begin{cases}\left(\frac{\pi \nu d^{3} D}{\ln \frac{4 E_{C}}{\delta}}\right)^{1 / 2} & T \ll D /(\mathcal{L} d), \\ \left(\frac{12 \nu d^{2} D^{2}}{T}\right)^{1 / 3} & T \gg D /(\mathcal{L} d) .\end{cases}
$$

Note in the high temperature limit $T \gg D /(\mathcal{L} d)$ the above results match with those derived earlier for metallic nanorings with the aid of different approaches 39,40 . On the other hand, at lower $T$ our results are different. 
This difference is due to low temperature saturation of $\tau_{\phi}$ which was not accounted for in 39,40 . A non-trivial feature predicted here is that - in contrast to weak localization 4 - the crossover from thermal to quantum dephasing is controlled by the ring perimeter $\mathcal{L}$. This is because only sufficiently long electron paths fully encircling the ring are sensitive to the magnetic flux and may contribute to $\mathrm{AB}$ oscillations of conductance.

We believe that the quantum dot rings considered here can be directly used for further experimental investigations of quantum coherence of interacting electrons in nanoscale conductors at low temperatures.

\section{COMPARISON WITH EXPERIMENTS AND CONCLUDING REMARKS}

Turning to the experimental situation in the field, it is important to emphasize again that low temperature saturation of the electron decoherence time has been repeatedly observed in numerous experiments and is presently considered as firmly established and indisputably existing phenomenon. Although in some cases this phenomenon can be attributed to various extrinsic mechanisms, like magnetic impurities, overheating etc., in the vast majority of cases none of such extrinsic mechanisms can reasonably account for experimental observations. On the other hand, it was demonstrated above that electronelectron interactions universally provide non-vanishing electron dephasing down to $T=0$ in all types of disordered conductors. Therefore, it would be interesting to perform quantitative comparison between our universal formula for $\tau_{\varphi 0}$, Eq. (103), and experimental values of the electron decoherence time measured in different structures.

Note that in some of our earlier publications $4,41,42$ we have already demonstrated a good quantitative agreement between our theoretical predictions $\underline{4}$ and experimental data for $\tau_{\varphi 0}$ obtained for numerous metallic wires and quasi-1d semiconductors. Here we address the experiments on quantum dot structures as well as on both weakly and highly disordered metals.

First turning to quantum dots, we recall that in all 14 samples reported in experiments with open quantum dots performed by different groups 8 , $43-46$ the values $\tau_{\varphi 0}$ were found to rather closely follow a simple dependence $\underline{\underline{46}}$

$$
\tau_{\varphi 0} \approx \tau_{D}
$$

This approximate scaling was observed within the interval of dwell times $\tau_{D}$ of about 3 decades, see Fig. 5 in느. Our Eq. (103) essentially reproduces this scaling, especially having in mind that the dimensionless conductance $g$ was of order one (or slighlty larger) in almost all samples $8.43-46$. To the best of our knowledge no alternative explanation for the scaling (131) has been offered until now. Thus, we conclude that our theory is clearly consistent with the available experimental data on zero temperature electron dephasing in open quantum dots.

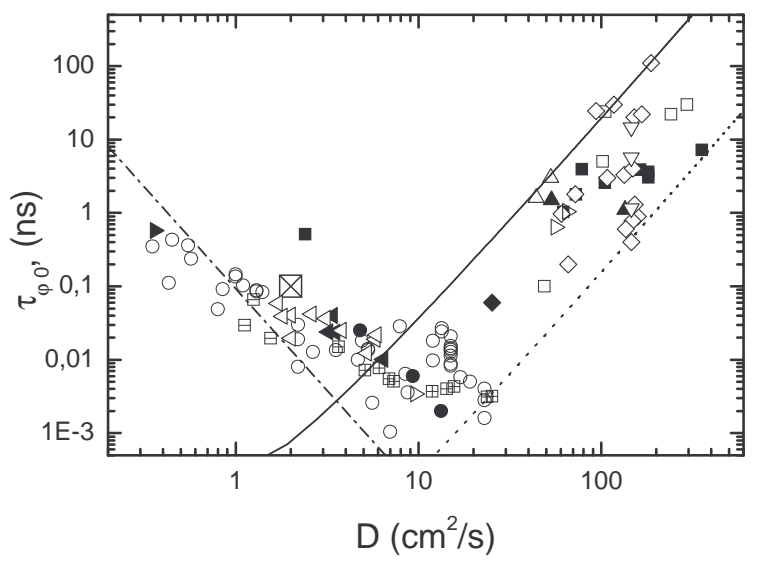

FIG. 13: The low temperature dephasing times observed in various experiments for the following samples: $\mathrm{Au}-1$ to $\mathrm{Au}-$ $6^{6}, \mathrm{Au}-7^{50}, \mathrm{Au}-8$ and $\mathrm{Au}-10^{51}(\mathbf{\square}) ; 44$ samples (AuPd and $\mathrm{AgPd}^{4}{ }^{47}(\mathrm{\circ}) ; 18$ samples${ }^{48}: \mathrm{Au}_{2} \mathrm{Al}(\triangleleft), \mathrm{Sb}(\triangleleft), \mathrm{Sc}_{85} \mathrm{Ag}_{15}(\bullet)$, $\mathrm{V}_{3} \mathrm{Al}(\boxminus) ; 9$ samples $(\mathrm{CuGeAu})^{49}(\boxplus) ; 15$ samples $(\mathrm{Au}, \mathrm{Ag}$ and $\mathrm{Cu})^{52}$ and $\mathrm{AgMI}^{5} \mathrm{~N}^{53}(\diamond) ; \mathrm{CF}-1$ and $\mathrm{CF}-2^{54}(\triangle) ; \mathrm{A}, \mathrm{B}$ $(\mathrm{Au})^{55}, \mathrm{Au}^{7}, \mathrm{Ag}^{56}$ and $\mathrm{Ag} 2^{57}(\square) ; \mathrm{S}, \mathrm{M}$ and $\mathrm{L}(\mathrm{Pt})^{58}(\bullet)$; $\mathrm{D}$ and $\mathrm{F}^{59}(\triangleright) ; \mathrm{Ag}, \mathrm{AgFe} 1$ and $\mathrm{AgFe}_{2}{ }^{60}(\nabla) ; 10$ samples ${ }^{61}$ within the box $(\otimes) ; 2(\mathrm{Au})^{62}$ and $1(\mathrm{Au})^{63}(\mathbf{\Delta})$; al- $1^{64}(\diamond)$. Our Eq. (111) for $\gamma=0.2$ and 1 is indicated respectively by solid and dashed lines, while Eq. (112) for $g_{c}=150$ is depicted by dashed-dotted line.

Let us now consider spatially extended disordered conductors. As our theory of dephasing by electron-electron interactions predicts a rather steep increase of $\tau_{\varphi 0}$ with the system diffusion coefficient $D$, for most weakly disordered metals as $\tau_{\varphi 0} \propto D^{3}$, we can conclude that for a large number of disordered conductors $\tau_{\varphi 0}$ strongly increases with increasing $D$. This trend is indeed quite obvious for relatively weakly disordered conductors. On the other hand, Lin and coworkers $9.47-49$ analyzed numerous experimental data for $\tau_{\varphi 0}$ obtained by various groups in rather strongly disordered conductors with $D \lesssim 10$ $\mathrm{cm}^{2} / \mathrm{s}$ and observed systematic decrease of $\tau_{\varphi 0}$ with increasing $D$. The data could be fitted by the dependence $\tau_{\varphi 0} \propto D^{-\alpha}$ with the power $\alpha \gtrsim 1$. This trend is clearly just the opposite to one observed in less disordered conductors with $D \gtrsim 10 \mathrm{~cm}^{2} / \mathrm{s}$.

In Fig. 13 we collected experimental data for $\tau_{\varphi 0}$ obtained in about 130 metallic samples with similar Fermi velocities and diffusion coefficients varying by $\sim 3$ decades, from $D \approx 0.3 \mathrm{~cm}^{2} / \mathrm{s}$ to $D \approx 350 \mathrm{~cm}^{2} / \mathrm{s}$. The data were taken from about 30 different publications listed in the figure caption. We see that the the measured values of $\tau_{\varphi 0}$ strongly depend on $D$. Furthermore, this dependence turns out to be non-monotonous: For relatively weakly disordered structures with $D \gtrsim 10 \mathrm{~cm}^{2} / \mathrm{s}$ $\tau_{\varphi 0}$ increases with increasing $D$, while for strongly disordered conductors with $D \lesssim 10 \mathrm{~cm}^{2} / \mathrm{s}$ the opposite trend takes place. In addition to the data points in Fig. 13 we indicate the dependencies $\tau_{\varphi 0}(D)$ (111) and (112) for two models $(a)$ and $(b)$ discussed above. 
We observe that for $D \gtrsim 10 \mathrm{~cm}^{2} / \mathrm{s}$ the data points clearly follow the scaling (111). Practically all data points remain within the strip between the two lines corresponding to Eq. (111) with $\gamma=1$ (dashed line) and $\gamma=0.2$ (solid line). On the other hand, for more disordered conductors with $D \lesssim 10 \mathrm{~cm}^{2} / \mathrm{s}$ the data are consistent with the scaling (112) obtained within the model (b). We would like to emphasize that theoretical curves (111) and (112) are presented in Fig. 13 with no fit parameters except for a geometry factor $\gamma$ for the first dependence and the value $g_{c} \approx 150$ for the second one. This value of $g_{c}$ was estimated from the crossover condition (113) with $D \sim 10 \mathrm{~cm}^{2} / \mathrm{s}$ and $\gamma \sim 1$.

Now let us consider the data for strongly disordered conductors with $D<10 \mathrm{~cm}^{2} / \mathrm{s}$. As we already pointed out, the agreement between the data and the dependence (112) predicted within our simple model $(b)$ is reasonable, in particular for samples with $D<3 \mathrm{~cm}^{2} / \mathrm{s}$. At higher diffusion coefficients most of the data points indicate a weaker dependence of $\tau_{\varphi 0}$ on $D$ which appears natural in the vicinity of the crossover to the dependence (111). The best fit for the whole range $0.3 \mathrm{~cm}^{2} / \mathrm{s}<D<10$ $\mathrm{cm}^{2} / \mathrm{s}$ is achieved with the function $\tau_{\varphi 0} \propto D^{-\alpha}$ with the power $\alpha \approx 1.5 \div 2$.

Thus, we conclude that our theory allows to qualitatively understand and explain seemingly contradicting dependencies of $\tau_{\varphi 0}$ on $D$ observed in weakly and strongly disordered conductors. While the trend "less disorder less decoherence" (111) for sufficiently clean conductors is quite obvious, the opposite trend "more disorder - less decoherence" in strongly disordered structures requires a comment. The latter dependence may indicate that with increasing disorder electrons spend more time in the areas with fluctuating in time but spatially uniform potentials. As we already discussed in the beginning of Sec. 3, such fluctuating potentials do not dephase and thus $\tau_{\varphi 0}$ gets effectively increased. In other words, in this case the corresponding dwell time $\tau_{D}$ in Eq. (103) becomes longer with increasing disorder and, hence, the electron decoherence time $\tau_{\varphi 0}$ does so too.

Note that since local conductance fluctuations increase with increasing disorder, several grains can form a cluster with internal inter-grain conductances strongly exceeding those at its edges. In this case fluctuating potentials remain almost uniform inside the whole cluster which will then play a role of an effective (bigger) grain/dot. Accordingly, the average volume of such "composite dots" $\mathcal{V} \propto 1 / \delta$ may grow with increasing disorder, electrons will spend more time in these bigger dots and, hence, the electron decoherence time (103) will increase.

The above comparison with experiments confirms that our previous quasiclassical results $\underline{4}$ for $\tau_{\varphi 0}$ are applicable to relatively weakly disordered structures with $D \gtrsim 10$ $\mathrm{cm}^{2} / \mathrm{s}$, while for conductors with stronger disorder different expressions for $\tau_{\varphi 0}$ (e.g., Eq. (112) ) should be used. Our analysis also allows to rule out scattering on magnetic impurities as a cause of low temperature saturation of $\tau_{\varphi}$. The latter mechanism can explain neither strong and non-trivial dependence of the electron decoherence time on $D$ nor even the level of dephasing observed in numerous experiments. E.g., in order to be able to attribute dephasing times as short as $\tau_{\varphi 0} \lesssim 10^{-12} \mathrm{~s}$ to magnetic impurities one needs to assume huge concentration of such impurities ranging from few hundreds to few thousands ppm which appears highly unrealistic, in particular for systems like carbon nanotubes, 2DEGs or quantum dots. Similar arguments were independently put forward by Lin and coworkers $\stackrel{47.49}{ }$.

Thus, although electron dephasing due to scattering on magnetic impurities is by itself an interesting issue, its role in low temperature saturation of $\tau_{\varphi}$ in disordered conductors is sometimes strongly overemphasized. Since the latter phenomenon has been repeatedly observed in all types of disordered conductors, the physics behind it should most likely be universal and fundamental. We believe - and have demonstrated here - that it is indeed the case: Zero temperature electron decoherence in all types of conductors discussed above is caused by electronelectron interactions.

This work was supported in part by RFBR grant 0902-00886.
${ }^{1}$ G. Bergmann, Phys. Rep. 107, 1 (1984).

2 S. Chakravarty and A. Schmid, Phys. Rep. 140, 193 (1986).

3 A.G. Aronov and Yu.V. Sharvin, Rev. Mod. Phys. 59, 755 (1987).

4 D.S. Golubev and A.D. Zaikin, Phys. Rev. Lett. 81, 1074 (1998); Phys. Rev. B 59, 9195 (1999); Phys. Rev. B 62, 14061 (2000); J. Low. Temp. Phys. 132, 11 (2003).

5 R.P. Feynman and A.R. Hibbs, Quantum Mechanics and Path Integrals (McGraw Hill, NY, 1965).

6 P. Mohanty, E.M.Q. Jariwala, and R.A. Webb, Phys. Rev. Lett. 78, 3366 (1997).

7 C. Bäuerle, F. Mallet, F. Shopfer, D. Mailly, G. Eska, and L. Saminadayar, Phys. Rev. Lett. 95, 266805 (2005).
${ }^{8}$ D.P. Pivin, A. Andresen, J.P. Bird, and D.K. Ferry, Phys. Rev. Lett. 82, 4687 (1999).

9 J.J. Lin and J.P. Bird, J. Phys. Condens. Matter 14, R501 (2002).

10 G. Schön and A.D. Zaikin, Phys. Rep. 198, 237 (1990).

11 A.D. Zaikin, Physica B 203, 255 (1994).

12 M. Büttiker, Phys. Rev. B 46, 12485 (1992).

13 C.W.J. Beenakker, Rev. Mod. Phys. 69, 731 (1997).

14 Yu.V. Nazarov, Phys. Rev. Lett. 82, 1245 (1999).

15 D.S. Golubev and A.D. Zaikin, Phys. Rev. Lett. 86, 4887 (2001).

16 M. Kindermann and Yu.V. Nazarov, Phys. Rev. Lett. 91, 136802 (2003).

17 A.V. Galaktionov, D.S. Golubev, and A.D. Zaikin, Phys. 
Rev. B 68, 085317 (2003); Phys. Rev. B 68, 235333 (2003).

18 D.S. Golubev and A.D. Zaikin, Phys. Rev. B 69, 075318 (2004).

19 D.S. Golubev and A.D. Zaikin, Phys. Rev. B 70, 165423 (2004).

${ }^{20}$ D.A. Bagrets and Yu.V. Nazarov, Phys. Rev. Lett. 94, 056801 (2005).

21 D.S. Golubev, A.V. Galaktionov, and A.D. Zaikin, Phys. Rev. B 72, 205417 (2005).

22 A. Kamenev and A. Andreev, Phys. Rev. B 60, 2218 (1999).

23 D.S. Golubev and A.D. Zaikin, Phys. Rev. B 74, 245329 (2006).

24 D.S. Golubev and A.D. Zaikin, New J. Phys. 10, 063027 (2008).

25 D.S. Golubev and A.D. Zaikin, Physica E 40, 32 (2007).

26 A.G. Semenov, D.S. Golubev, and A.D. Zaikin, Phys. Rev. $B$ 79, 115302 (2009).

27 A.G. Semenov and A.D. Zaikin, Physica E 42, 600 (2010).

28 Yu.V. Nazarov, Superlattices Microstruct. 25, 1221 (1994).

29 B.L. Altshuler, A.G. Aronov, and D.E. Khmelnitskii, J. Phys. C 15, 7367 (1982).

30 C.L. Kane, R.A. Serota, and P.A. Lee, Phys. Rev. B 37, 6701 (1998).

31 N. Argaman, Phys. Rev. B 53, 7035 (1996).

32 P.W. Brouwer and C.W.J. Beenakker, J. Math. Phys. 37, 4904 (1996).

${ }^{33}$ G. Campagnano and Yu.V. Nazarov, Phys. Rev. B 74, 125307 (2006).

34 P.A. Mello, Phys. Rev. Lett. 60, 1089 (1988); P.A. Mello and A.D. Stone, Phys. Rev. B 44, 3559 (1991).

35 N. Argaman, Phys. Rev. Lett. 75, 2750 (1995).

36 P.W. Brouwer, A. Lamacraft, and K. Flensberg, Phys. Rev. $B$ 72, 075316 (2005).

37 S.V. Panyukov and A.D. Zaikin, Phys. Rev. Lett. 67, 3168 (1991); J. Low Temp. Phys. 73, 1 (1998).

38 G.L. Ingold and Yu.V. Nazarov. In: Single Charge Tunneling, ed. by H. Grabert and M.H. Devoret, NATO ASI Series B 294 (Plenum Press, New York 1992) p. 21.

39 C. Texier and G. Montambaux, Phys. Rev. B 72, 115327 (2005).

40 T. Ludwig and A.D. Mirlin, Phys. Rev. B 69, 193306 (2004).

41 D.S. Golubev and A.D. Zaikin, Physica B 255, 164 (1998).

42 D.S. Golubev, A.D. Zaikin, and G. Schön, J. Low Temp. Phys. 126, 1355 (2002).

43 J.P. Bird, K. Ishibashi, D.K. Ferry, Y. Ochiai, Y. Aoyagi, and T. Sugano, Phys. Rev. B 51, 18037 (1995).
44 R.M. Clarke, I.H. Chan, C.M. Marcus, C.I. Duruoz, J.S. Harris, K. Campman, and A.C. Gossard, Phys. Rev. B 52, 2656 (1995).

45 A.G. Huibers, J.A. Folk, S.R. Patel, C.M. Marcus, C.I. Duruoz, and J.S. Harris, Phys. Rev. Lett. 83, 5090 (1999).

46 B. Hackens, S. Faniel, C. Gustin, X. Wallart, S. Bollaert, A. Cappy, and V. Bayot, Phys. Rev. Lett. 94, 146802 (2005).

47 J.J. Lin, T.C. Lee and S.W. Wang, Physica E 40, 25 (2007), see also further references therein.

48 J.J. Lin and L.Y. Kao, J. Phys.: Condens. Matter 13, L119 (2001).

49 S.M. Huang, H. Akimoto, K. Kono, and J.J. Lin, Phys. Rev. Lett. 99, 046601 (2007).

50 R.A. Webb, P. Mohanty, and E.M.Q. Jariwala, Fortsch. Phys. 46, 779 (1998).

51 P. Mohanty, private communication (2003).

${ }^{52}$ F. Pierre, A.B. Gougam, A. Anthore, H. Pothier, D. Esteve, and N.O. Birge, Phys. Rev. B 68, 085413 (2003).

53 N. Birge, private communication (2003).

${ }^{54}$ P. Mohanty and R.A. Webb, Phys. Rev. Lett. 91, 066604 (2003).

${ }^{55}$ F. Schopfer, C. Bäuerle, W. Rabaud, and L. Saminadayar, Phys. Rev. Lett. 90, 056801 (2003).

56 L. Saminadayar, P. Mohanty, R.A. Webb, P. Degiovanni, and C. Bäuerle, Physica E 40, 12 (2007).

57 F. Mallet, J. Ericsson, D. Mailly, S. Unlubayir, D. Reuter, A. Melnikov, A.D. Wieck, T. Micklitz, A. Rosch, T.A. Costi, L. Saminadayar, and C. Bäuerle, Phys. Rev. Lett. 97, 226804 (2006).

58 J.F. Lin, J.P. Bird, L. Rotkina, P.A. Bennett, Appl. Phys. Lett. 82, 802 (2003); J.F. Lin, J.P. Bird, L. Rotkina, Physica E 19, 112 (2003).

59 A. Trionfi, S. Lee, and D. Natelson, Phys. Rev. B 72, 035407 (2005).

60 G.M. Alzoubi and N.O. Birge, Phys. Rev. Lett. 97, 226803 (2006).

61 A. Sahnoune, J.O. Strom-Olsen, and H.E. Fisher, Phys. Rev. B 46, 10035 (1992).

62 P.M. Echternach, M.E. Gershenson, and H.M. Bozler, Phys Rev. B 47, 13659 (1993).

63 P.M. Echternach, M.E. Gershenson, H.M. Bozler, A.L. Bogdanov, and B. Nilsson, Phys. Rev. B 48, 11516 (1993).

${ }^{64}$ F. Altomare, A.M. Chang, M.R. Melloch, Y. Hong, and C.W. Tu, Appl. Phys. Lett. 86, 172501 (2005), Erratum, cond-mat/0412210 